\title{
REPRESENTATION AND APPROXIMATION OF AMBIT FIELDS IN HILBERT SPACE
}

\author{
FRED ESPEN BENTH AND HEIDAR EYJOLFSSON
}

\begin{abstract}
We lift ambit fields as introduced by Barndorff-Nielsen and Schmiegel [5] to a class of Hilbert space-valued volatility modulated Volterra processes. We name this class Hambit fields, and show that they can be expressed as a countable sum of weighted real-valued volatility modulated Volterra processes. Moreover, Hambit fields can be interpreted as the boundary of the mild solution of a certain first order stochastic partial differential equation. This stochastic partial differential equation is formulated on a suitable Hilbert space of functions on the positive real line with values in the state space of the Hambit field. We provide an explicit construction of such a space. Finally, we apply this interpretation of Hambit fields to develop a finite difference scheme, for which we prove convergence under some Lipschitz conditions.
\end{abstract}

\section{INTRODUCTION}

Ambit fields, introduced by Barndorff-Nielsen and Schmiegel [5], have attracted much attention in recent years being a powerful tool to model stochastic phenomena like turbulence, tumor growth, weather dynamics, and financial prices (see Barndorff-Nielsen and Schmiegel [5], Barndorff-Nielsen, Benth and Veraart [2, 3], Benth and Šaltyte Benyth [7], Corcuera et al. [14] and Vedel Jensen et al. [22]). The class of ambit fields is analytically tractable, and provides a framework for a probabilistic description of the dynamics of noisy systems which are more general than the conventional stochastic partial differential equations (see Barndorff-Nielsen, Benth and Veraart [1]).

Following Barndorff-Nielsen and Schmiegel [5], an ambit field is defined as a real-valued random field on $\mathbb{R}_{+} \times \mathbb{R}^{d}$ and a filtered probability space $\left(\Omega, \mathcal{F},\left\{\mathcal{F}_{t}\right\}_{t \geq 0}, P\right)$ of the form

$$
Z(t, x)=\int_{0}^{t} \int_{A} g(t, s, x, y) \sigma(s, y) L(d y, d s) .
$$

Here, $(t, x) \in \mathbb{R}_{+} \times A, A \subset \mathbb{R}^{d}$ is a Borel measurable subset called the ambit set, $g$ a measurable realvalued function on $\mathbb{R}_{+} \times \mathbb{R}_{+} \times \mathbb{R}^{d} \times \mathbb{R}^{d}$ and $\sigma$ a real-valued predictable random field on $\mathbb{R}_{+} \times \mathbb{R}^{d}$. The function $g$ is sometimes referred to as the kernel function, and $\sigma$ is modelling the volatility or intermittency. Finally, $L$ is a Lévy basis, where $\sigma$ and $L$ are assumed independent. In this paper we restrict our attention to $L$ being a square-integrable Lévy basis. Moreover, we suppose $L$ to have mean zero. Using the integration concept of Walsh (see Walsh [23]), the ambit field $Z(t, x)$ in (1.1) is well-defined if

$$
\int_{[0, t] \times A} g^{2}(t, s, x, y) \mathbb{E}\left[\sigma^{2}(s, y)\right] \operatorname{Var}\left(L^{\prime}(y, s)\right) c(d y, d s)<\infty,
$$

where $c$ is the control measure and $L^{\prime}$ the Lévy seed associated with $L$. Indeed, $\operatorname{Var}\left(L^{\prime}(x, t)\right) c(d x, d t)$ is equal to the Radon-Nikodym derivative of the covariance measure of $L$. We refer to Barndorff-Nielsen and Schmiegel [5] or, the more recent survey paper of Barndorff-Nielsen, Benth and Veraart [4] for details and discussions about ambit fields and their properties and applications. An analysis on stochastic integration for random fields as introduced by Walsh applied to ambit fields can be found in Barndorff-Nielsen, Benth and Veraart [1]. Note that we consider the ambit field $Z$ without drift and restrict our attention to times $t$ which are positive. Moreover, in the general definition of ambit fields by Barndorff-Nielsen and Schmiegel [5], the ambit set $A$ is also allowed to be dependent on time and space $(t, x)$. We refrain from such generality here, as in most cases such dependency can be included in the specification of the kernel function $g$.

Date: July 16, 2018.

F. E. Benth acknowledges financial support from the research projects "Managing Weather Risk in Energy Markets (MAWREM)" and "Finance, Insurance, Energy, Weather and Stochastics (FINEWSTOCH)", both funded by the Norwegian Research Council. H. Eyjolfsson acknowledges financial support from Finansmarkedsfondet. 
The objective of this paper is to define a class of Volterra processes with values in Hilbert space which provides an infinite-dimensional formulation of ambit fields. We shall call these processes Hambit fields, referring to the Hilbert space-valued structure. After defining Hambit fields, we discuss some specific examples and relate the Hambit fields to the "classical" ambit fields $Z(t, x)$ as in (1.1). Under mild conditions, we can compute a rather explicit expression for the characteristic functional of a Hambit field. If $L$ is a Wiener basis, then the Hambit field becomes a conditional Gaussian Hilbert-valued random variable.

One of our main results is the representation of Hambit fields as a weighted series of volatility modulated Volterra processes. Volatility modulated Volterra processes generalize Lévy semistationary processes, for which Ornstein-Uhlenbeck processes constitute a particular case. Lévy semistationary processes have been applied to model energy spot prices (see Barndorff-Nielsen, Benth and Veraart [2]), while in BarndorffNielsen, Benth and Veraart [3] ambit fields have been proposed as a model for energy forward markets. Thus, the representation of Hambit fields in terms of a weighted series of volatility modulated Volterra processes provides us with a useful theoretical link between spot and forward market models based on ambit fields. This result shows the power of lifting ambit fields to Hilbert space, which gives a simple approach to show such a representation using basis function expansions. For an extensive discussion of energy spot and forward markets and multi-factor commodity pricing models, we refer to Benth, Šaltyte Benth and Koekebakker[8].

Hambit fields can be seen as a Volterra process in Hilbert space. By a simple splitting of time in the integration and in the kernel function they can be viewed as mild solutions of a first order stochastic partial differential equation formulated in a Hilbert space of functions from $\mathbb{R}_{+}$into the state space of the Hambit field. We construct an explicit space of such functions on $\mathbb{R}_{+}$, generalizing the Filipovic space of real-valued absolutely continuous functions on $\mathbb{R}_{+}$(see Filipovic [17]). Via an evaluation map, we can transform the solution of the stochastic partial differential equation linearly into a Hambit field. This result follows from a commutativity property of the stochastic integral with linear maps.

Using the interpretation of Hambit fields as the boundary solution of a stochastic partial differential equations, we develop an iterative finite difference scheme. The scheme is formulated in the state space of the Hambit field, and under certain Lipschitz conditions on the kernel function the convergence rate of the scheme is controlled. Our results provide a framework for numerical studies of ambit fields, taking a different route than the Fourier-based method suggested by Eyjolfsson [16].

Our results are presented as follows. In the next section we define Hambit fields and study some elementary aspects and develop a series representation in terms of volatility modulated Volterra processes. We proceed in Section 3 by introducing a stochastic partial differential equation for which we can relate Hambit fields as a boundary solution. Finally, Section 4 is devoted to the development and analysis of a finite difference scheme for this stochastic partial differential equation.

\section{DEFINITION AND ANALYSIS OF HAMBit FIELDS}

In this Section we introduce a class Hilbert-space valued Volterra processes that provides a general definition of ambit fields as defined in 1.1.

In the sequel, we shall operate with the three separable Hilbert spaces $\mathcal{U}, \mathcal{V}$ and $\mathcal{H}$, where we denote the respective inner products by $(\cdot, \cdot)_{i}$ and corresponding norms $|\cdot|_{i}, i=\mathcal{U}, \mathcal{V}, \mathcal{H}$. Let $t \mapsto \sigma(t)$ be a $\mathcal{U}$ valued predictable stochastic process. Introduce the measurable function $\Gamma: \mathbb{R}_{+}^{2} \rightarrow \mathcal{L}(\mathcal{U}, \mathcal{L}(\mathcal{V}, \mathcal{H}))$, where $\mathcal{L}(\mathcal{V}, \mathcal{H})$ is the space of bounded operators from $\mathcal{V}$ to $\mathcal{H}$, and $\mathcal{L}(\mathcal{U}, \mathcal{L}(\mathcal{V}, \mathcal{H}))$ the space of bounded operators from $\mathcal{U}$ to $\mathcal{L}(\mathcal{V}, \mathcal{H})$. Note that since $\mathcal{H}$ is a Hilbert space, $\mathcal{L}(\mathcal{V}, \mathcal{H})$ becomes a Banach space, which again implies that $\mathcal{L}(\mathcal{U}, \mathcal{L}(\mathcal{V}, \mathcal{H}))$ is a Banach space under respective operator norms. By the predictability of the process $\sigma$, we find that $s \in[0, t] \mapsto \Gamma(s, t)(\sigma(s)) \in \mathcal{L}(\mathcal{V}, \mathcal{H})$ is predictable. Finally, assume that $L$ is a square-integrable $\mathcal{V}$-valued Lévy process with zero mean (i.e., $L$ is a martingale). Denote by $\mathcal{Q} \in \mathcal{L}(\mathcal{V})$ the covariance operator of $L$, being a symmetric, non-negative definite trace class operator. Note that we use the notation $\mathcal{L}(\mathcal{V})$ for $\mathcal{L}(\mathcal{V}, \mathcal{V})$, and that we do not assume independence between $\sigma$ and $L$.

We define a Hambit field as follows:

Definition 2.1. Suppose, for each $t \leq T$,

$$
\mathbb{E}\left[\int_{0}^{t}\left\|\Gamma(t, s)(\sigma(s)) \mathcal{Q}^{1 / 2}\right\|_{H S}^{2} d s\right]<\infty,
$$


where $\|\cdot\|_{H S}$ denotes the Hilbert-Schmidt norm on $\mathcal{L}(\mathcal{V}, \mathcal{H})$. Then the $\mathcal{H}$-valued stochastic process $\{X(t)\}_{t \in[0, T]}$ defined as

$$
X(t)=\int_{0}^{t} \Gamma(t, s)(\sigma(s)) d L(s)
$$

is called a Hambit field.

We remark that by Peszat and Zabczyk [20, Sect. 8.6], the conditions on $\Gamma$ and $\sigma$ make the stochastic integral with respect to $L$ well-defined, in fact the following isometry holds

$$
\mathbb{E}\left[|X(t)|_{\mathcal{H}}^{2}\right]=\mathbb{E}\left[\int_{0}^{t}\left\|\Gamma(t, s)(\sigma(s)) \mathcal{Q}^{1 / 2}\right\|_{\mathrm{HS}}^{2} d s\right] .
$$

A convenient sufficient condition for $(2.1)$ is formulated in the next Lemma:

Lemma 2.2. Suppose for each $t \leq T$ that

$$
\int_{0}^{t}\|\Gamma(t, s)\|_{o p}^{2} \mathbb{E}\left[|\sigma(s)|_{\mathcal{U}}^{2}\right] d s<\infty
$$

then Condition (2.1) holds. Here, $\|\cdot\|_{\text {op }}$ denotes the operator norm in $\mathcal{L}(\mathcal{U}, \mathcal{L}(\mathcal{V}, \mathcal{H}))$.

Proof. If $\left\{v_{m}\right\}_{m \in \mathbb{N}}$ is an ONB in $\mathcal{V}$, then by definition of the Hilbert-Schmidt norm and $\Gamma(t, s)(\sigma(s)) \in$ $L(\mathcal{V}, \mathcal{H})$ yield

$$
\begin{aligned}
\left\|\Gamma(t, s)(\sigma(s)) \mathcal{Q}^{1 / 2}\right\|_{\mathrm{HS}}^{2} & =\sum_{m=1}^{\infty}\left|\Gamma(t, s)(\sigma(s)) \mathcal{Q}^{1 / 2} v_{m}\right|_{\mathcal{V}}^{2} \\
& \leq\|\Gamma(t, s)(\sigma(s))\|_{\mathrm{op}}^{2} \sum_{m=1}^{\infty}\left|\mathcal{Q}^{1 / 2} v_{m}\right|_{\mathcal{V}}^{2} \\
& \leq\|\Gamma(t, s)\|_{\mathrm{op}}^{2}|\sigma(s)|_{\mathcal{U}}^{2}\left\|\mathcal{Q}^{1 / 2}\right\|_{\mathrm{HS}}^{2} .
\end{aligned}
$$

Since $Q$ is trace class operator, the result follows.

We note that this sufficient condition on the integrability of the "kernel function" $\Gamma$ and the "volatility" $\sigma$ share some similarity with the analogous condition for classical ambit fields (see (1.2)).

Let us look an example of a Hambit field motivated by the analysis of Benth, Rüdiger and Süss [12]. Consider a stochastic volatility modulated Ornstein-Uhlenbeck process of the following form:

$$
d X(t)=\mathcal{A} X(t) d t+\sigma(t) d W(t), \quad X(0)=X_{0} \in \mathcal{H},
$$

where $\mathcal{A}$ is a (possibly unbounded) linear operator on $\mathcal{H}$ which is densely defined and generating a $C_{0^{-}}$ semigroup $\mathcal{S}$. Moreover, it is assumed that $W$ is an $\mathcal{H}$-valued Wiener process with covariance operator $\mathcal{Q}$. Hence, we choose $\mathcal{V}=\mathcal{H}$. The volatility process $\sigma(t)$ is assumed to be predictable and take values in the space of Hilbert-Schmidt operators on $\mathcal{H}$, denoted $\mathcal{L}_{\mathrm{HS}}(\mathcal{H})$. Thus, we let $\mathcal{U}=\mathcal{L}_{\mathrm{HS}}(\mathcal{H})$, and recall that whenever $\mathcal{H}$ is a separable Hilbert space, $\mathcal{L}_{\mathrm{HS}}(\mathcal{H})$ becomes a separable Hilbert space under the HilbertSchmidt norm. A mild solution of (2.3) is

$$
X(t)=\mathcal{S}_{t} X_{0}+\int_{0}^{t} \mathcal{S}_{t-s} \sigma(s) d W(s)
$$

Note that the stochastic integral is well-defined as long as we have

$$
\mathbb{E}\left[\int_{0}^{t}\left\|\mathcal{S}_{t-s} \sigma(s) \mathcal{Q}^{1 / 2}\right\|_{\mathrm{HS}}^{2} d s\right]<\infty .
$$

Now, define $\Gamma(t, s) \in \mathcal{L}\left(\mathcal{L}_{\mathrm{HS}}(\mathcal{H})\right)$ as $\Gamma(t, s): \sigma \mapsto \mathcal{S}_{t-s} \sigma$. For any $\sigma \in \mathcal{L}_{\mathrm{HS}}(\mathcal{H}), \mathcal{S}_{t-s} \sigma$ becomes a linear bounded operator on $\mathcal{H}$, and since $\sigma$ is Hilbert-Schmidt, it follows that $\mathcal{S}_{t-s} \sigma$ is Hilbert-Schmidt as well. Hence, $\Gamma(t, s)$ maps linearly the Hilbert-Schmidt operators on $\mathcal{H}$ into itself. Moreover, since we have

$$
\|\Gamma(t, s)\|_{\mathrm{op}}=\sup _{\|\sigma\|_{\mathrm{Hs}} \leq 1}\|\Gamma(t, s)(\sigma)\|_{\mathrm{HS}}=\sup _{\|\sigma\|_{\mathrm{Hs}} \leq 1}\left\|\mathcal{S}_{t-s} \sigma\right\|_{\mathrm{HS}} \leq\left\|\mathcal{S}_{t-s}\right\|_{\mathrm{op}} \sup _{\|\sigma\|_{\mathrm{Hs}} \leq 1}\|\sigma\|_{\mathrm{HS}}
$$


and therefore $\|\Gamma(t, s)\|_{\text {op }} \leq\left\|\mathcal{S}_{t-s}\right\|_{\text {op }}<\infty$. By the general exponential growth bound on a $C_{0}$-semigroup and norm estimates on the Hilbert-Schmidt norm, we find

$$
\left\|\Gamma(t, s)(\sigma(s)) \mathcal{Q}^{1 / 2}\right\|_{\mathrm{HS}}^{2}=\left\|\mathcal{S}_{t-s} \sigma(s) \mathcal{Q}^{1 / 2}\right\|_{\mathrm{HS}}^{2} \leq\left\|Q^{1 / 2}\right\|_{\mathrm{op}}^{2} M \mathrm{e}^{w(t-s)}\|\sigma(s)\|_{\mathrm{HS}}^{2}
$$

for positive constants $M$ and $w$. But then, according to Lemma 2.2, it is sufficient that

$$
\int_{0}^{t} \mathbb{E}\left[\|\sigma(s)\|_{\mathrm{HS}}^{2}\right] d s<\infty,
$$

to ensure integrability. Thus, we conclude that the stochastic integral in $X$ defined in (2.4) is a Hambit field.

In Benth, Rüdiger and Süss [12] a particular definition of the stochastic volatility process $\sigma$ is considered. Indeed, they propose a generalization of the BNS stochastic volatility model (see Barndorff-Nielsen and Shephard [6]) to operator-valued Ornstein-Uhlenbeck (OU) processes. To this end, let $\mathcal{Y}(t)$ be a symmetric non-negative definite process with values in $\mathcal{L}_{\mathrm{HS}}(\mathcal{H})$ defined by the dynamics

$$
d \mathcal{Y}(t)=\mathbb{C} \mathcal{Y}(t) d t+d \mathcal{Z}(t)
$$

where $\mathcal{Z}(t)$ is an $\mathcal{L}_{\mathrm{HS}}(\mathcal{H})$-valued square integrable Lévy process and $\mathbb{C} \in \mathcal{L}\left(\mathcal{L}_{\mathrm{HS}}(\mathcal{H})\right)$. Under suitable conditions on $\mathbb{C}$ and $\mathcal{Z}$ we can ensure that $\mathcal{Y}(t)$ is a symmetric, non-negative definite Hilbert-Schmidt operator (see Benth, Rüdiger and Süss [12] for details). Moreover, following the arguments in Prop. 3.1 of Benth, Rüdiger and Süss [12], we can show that

$$
\operatorname{Tr}(\mathcal{Y}(t))=\operatorname{Tr}\left(\mathrm{e}^{\mathbb{C} t} \mathcal{Y}_{0}\right)+\operatorname{Tr}\left(\int_{0}^{t} \mathrm{e}^{\mathbb{C} s} d s \mathbb{E}[\mathcal{Z}(1)]\right),
$$

and

$$
\mathbb{E}\left[\|\sigma(t)\|_{\mathrm{HS}}^{2}\right]=\sum_{k=1}^{\infty}\left(\sigma^{2}(t) h_{k}, h_{k}\right)_{\mathcal{H}}=\operatorname{Tr}(\mathcal{Y}(t)) .
$$

Thus, as the $C_{0}$-semigroup $\exp (\mathbb{C} t)$ of $\mathbb{C}$ is Bochner integrable since $\mathbb{C}$ is bounded, and $\mathcal{Z}(1)$ has finite expected value, it follows from the continuity of the Bochner integral that $t \mapsto \operatorname{Tr}(\mathcal{Y}(t))$ is integrable on finite time intervals. This shows that we can use $\mathcal{Y}^{1 / 2}(t)$ as a stochastic volatility process $\sigma$ in the definition of a Hambit field.

Let us return back to the general discussion of Hambit fields. Our next result concerns the $L^{2}$-proximity of two distinct Hambit fields.

Lemma 2.3. Suppose the Hambit fields

$$
X_{i}(t)=\int_{0}^{t} \Gamma_{i}(t, s)\left(\sigma_{i}(s)\right) d L(s), \quad i=1,2,
$$

fulfill the premise of Lemma2.2 Then,

$$
\begin{aligned}
\mathbb{E}\left[\left|X_{1}(t)-X_{2}(t)\right|_{\mathcal{H}}^{2}\right] \leq & \left\|\mathcal{Q}^{1 / 2}\right\|_{H S}^{2} \int_{0}^{t}\left\|\Gamma_{1}(t, s)-\Gamma_{2}(t, s)\right\|_{o p}^{2} \mathbb{E}\left[\left|\sigma_{1}(s)\right|_{\mathcal{U}}^{2}\right] d s \\
& +\left\|\mathcal{Q}^{1 / 2}\right\|_{H S}^{2} \int_{0}^{t}\left\|\Gamma_{2}(t, s)\right\|_{o p}^{2} \mathbb{E}\left[\left|\sigma_{1}(s)-\sigma_{2}(s)\right|_{\mathcal{U}}^{2}\right] d s
\end{aligned}
$$

for all $t \geq 0$.

Proof. By the identity

$$
\Gamma_{1}(t, s)\left(\sigma_{1}(s)\right)-\Gamma_{2}(t, s)\left(\sigma_{2}(s)\right)=\left(\Gamma_{1}(t, s)-\Gamma_{2}(t, s)\right)\left(\sigma_{1}(s)\right)+\Gamma_{2}(t, s)\left(\sigma_{1}(s)-\sigma_{2}(s)\right),
$$

the isomety (2.2) and (the proof of) Lemma 2.2, it holds that

$$
\mathbb{E}\left[\left|\int_{0}^{t}\left(\Gamma_{1}(t, s)-\Gamma_{2}(t, s)\right)\left(\sigma_{1}(s)\right) d L(s)\right|_{\mathcal{H}}^{2}\right] \leq\left\|\mathcal{Q}^{1 / 2}\right\|_{\mathrm{HS}}^{2} \int_{0}^{t}\left\|\Gamma_{1}(t, s)-\Gamma_{2}(t, s)\right\|_{\mathrm{op}}^{2} \mathbb{E}\left[\left|\sigma_{1}(s)\right|_{\mathcal{U}}^{2}\right] d s,
$$

and

$$
\mathbb{E}\left[\left|\int_{0}^{t} \Gamma_{2}(t, s)\left(\sigma_{1}(s)-\sigma_{2}(s)\right) d L(s)\right|_{\mathcal{H}}^{2}\right] \leq\left\|\mathcal{Q}^{1 / 2}\right\|_{\mathrm{HS}}^{2} \int_{0}^{t}\left\|\Gamma_{2}(t, s)\right\|_{\mathrm{op}}^{2} \mathbb{E}\left[\left|\sigma_{1}(s)-\sigma_{2}(s)\right|_{\mathcal{U}}^{2}\right] d s
$$


from which the conclusion follows.

As an application of the above result, we consider approximating a given Hambit field as follows: let $\Pi_{n}:=\left\{s_{i}\right\}_{i=1}^{n}, n=1,2, \ldots$, be a sequence of partitions of $[0, t]$ such that $\max _{1 \leq i \leq n-1}\left|s_{i+1}-s_{i}\right| \downarrow 0$. Let, for each $n \in \mathbb{N}$,

$$
\Gamma_{n}(t, s):=\sum_{i=1}^{n-1} \Gamma\left(t, s_{i}\right) 1_{\left(s_{i}, s_{i+1}\right]}(s), \text { and } \sigma_{n}(s):=\sum_{i=1}^{n-1} \sigma\left(s_{i}\right) 1_{\left(s_{i}, s_{i+1}\right]}(s),
$$

be the corresponding piecewise constant approximations of $\Gamma(t, \cdot)$ and $\sigma(\cdot)$ on $[0, t]$. Then, it follows by Lemma 2.3 that the Hambit fields

$$
X_{n}(t):=\int_{0}^{t} \Gamma_{n}(t, s)\left(\sigma_{n}(s)\right) d L(s)
$$

for $n \in \mathbb{N}$ approximate the original Hambit field, $X(t)$, if

$$
\int_{0}^{t}\left(\left\|\Gamma(t, s)-\Gamma_{n}(t, s)\right\|_{\mathrm{op}}^{2} \mathbb{E}\left[|\sigma(s)|_{\mathcal{U}}^{2}\right]+\left\|\Gamma_{n}(t, s)\right\|_{\mathrm{op}}^{2} \mathbb{E}\left[\left|\sigma(s)-\sigma_{n}(s)\right|_{\mathcal{U}}^{2}\right]\right) d s \rightarrow 0,
$$

when $n \rightarrow \infty$. Note that the approximative Hambit fields given by (2.7) are well defined if (2.8) holds. Indeed, it follows by (2.8) and Lemma 2.3 that $\mathbb{E}\left[\left|X(t)-X_{n}(t)\right|_{\mathcal{H}}^{2}\right] \rightarrow 0$ when $n \rightarrow \infty$, which in turn means that $\lim _{n \rightarrow \infty} \mathbb{E}\left[\left|X_{n}(t)\right|_{\mathcal{H}}^{2}\right]=\mathbb{E}\left[|X(t)|_{\mathcal{H}}^{2}\right]$. For future reference we state the above convergence condition in an assumption.

Assumption 1. A Hambit field $X(t)$ can be piecewise constantly approximated if condition (2.8) is fulfilled, where $\Gamma_{n}(t, s)$ and $\sigma_{n}(s)$ are defined by (2.6) and the limit is obtained by taking finer and finer partitions.

We remark that the purpose of the above assumption is to identify conditions under which

$$
\mathbb{E}\left[\left|X(t)-X_{n}(t)\right|_{\mathcal{H}}\right] \rightarrow 0,
$$

as we consider finer and finer partitions. Recall that the stochastic integral defining a Hambit field is built by first defining it for simple functions, and then extending it via the isometric formula (2.2), which means that the simple functions are dense in the space of integrable functions. If the integrand $s \mapsto \Gamma(t, s)(\sigma(s))$ is continuous function from $[0, t]$ into the space of bounded linear operators with norm defined by $\left\|\cdot \mathcal{Q}^{1 / 2}\right\|_{\mathrm{HS}}$, then one can choose the simple functions as in (2.6), and (2.9) follows by the isometric formula (2.2).

Suppose $s \mapsto \Gamma(t, s)$ is continuous with respect to $\|\cdot\|_{\text {op }}$ on $s \in[0, t]$, and assume $\sup _{s \in[0, t]} \mathbb{E}\left[|\sigma(s)|_{\mathcal{U}}^{2}\right]<$ $\infty$ and

$$
\int_{0}^{t}\|\Gamma(t, s)\|_{\mathrm{op}}^{2} \mathbb{E}\left[\left|\sigma(s)-\sigma_{n}(s)\right|_{\mathcal{U}}^{2} d s\right] \rightarrow 0
$$

when $n \rightarrow 0$. Then, Assumption 1 holds. Indeed, by the triangle inequality

$$
\begin{aligned}
\int_{0}^{t}\left\|\Gamma_{n}(t, s)\right\|_{\mathrm{op}}^{2} \mathbb{E}\left[\left|\sigma(s)-\sigma_{n}(s)\right|_{\mathcal{U}}^{2}\right] d s \\
\leq 2 \int_{0}^{t}\left\|\Gamma(t, s)-\Gamma_{n}(t, s)\right\|_{\mathrm{op}}^{2} \mathbb{E}\left[\left|\sigma(s)-\sigma_{n}(s)\right|_{\mathcal{U}}^{2}\right] d s \\
\quad+2 \int_{0}^{t}\|\Gamma(t, s)\|_{\mathrm{op}}^{2} \mathbb{E}\left[\left|\sigma(s)-\sigma_{n}(s)\right|_{\mathcal{U}}^{2}\right] d s .
\end{aligned}
$$

By assumption, the second term above converges to zero as $n \rightarrow 0$. Consider the first term: Note that

$$
\begin{aligned}
\sup _{s \in[0, t]} \mathbb{E}\left[\left|\sigma(s)-\sigma_{n}(s)\right|_{\mathcal{U}}^{2}\right] & \leq 2 \sup _{s \in[0, t]} \mathbb{E}\left[|\sigma(s)|_{\mathcal{U}}^{2}\right]+2 \sup _{s \in[0, t]} \mathbb{E}\left[\left|\sigma_{n}(s)\right|_{\mathcal{U}}^{2}\right] \\
& \leq 4 \sup _{s \in[0, t]} \mathbb{E}\left[|\sigma(s)|_{\mathcal{U}}^{2}\right]
\end{aligned}
$$

since

$$
\sup _{s \in[0, t]} \mathbb{E}\left[\left|\sigma_{n}(s)\right|_{\mathcal{U}}^{2}\right]=\sup _{s_{i} \in \Pi_{n}} \mathbb{E}\left[\left|\sigma\left(s_{i}\right)\right|_{\mathcal{U}}^{2}\right] \leq \sup _{s \in[0, t]} \mathbb{E}\left[|\sigma(s)|_{\mathcal{U}}^{2}\right]
$$


Hence,

$$
\begin{array}{rl}
\int_{0}^{t}\left\|\Gamma(t, s)-\Gamma_{n}(t, s)\right\|_{\mathrm{op}}^{2} & \mathbb{E}\left[\left|\sigma(s)-\sigma_{n}(s)\right|_{\mathcal{U}}^{2}\right] d s \\
\leq & \sup _{s \in[0, t]}\left\|\Gamma(t, s)-\Gamma_{n}(t, s)\right\|_{\mathrm{op}}^{2} \int_{0}^{t} \mathbb{E}\left[\left|\sigma(s)-\sigma_{n}(s)\right|_{\mathcal{U}}^{2}\right] d s \\
\leq & 4 t \sup _{s \in[0, t]} \mathbb{E}\left[|\sigma(s)|_{\mathcal{U}}^{2}\right] \sup _{s \in[0, t]}\left\|\Gamma(t, s)-\Gamma_{n}(t, s)\right\|_{\mathrm{op}}^{2},
\end{array}
$$

which tends to zero when $n \rightarrow \infty$ by uniform continuity. In conclusion, for these particular regularity conditions on $\Gamma$ and $\sigma$ we are ensured that Assumption 1 holds. This case is particularly relevant when $\Gamma(t, s)$ is equal to a $C_{0}$-semigroup, $\Gamma(t, s)=\mathcal{S}_{t-s}$.

In the next Proposition we present the characteristic functional of the Hambit field:

Proposition 2.4. Suppose that Assumption 1 holds and assume that $\sigma$ is independent of L. Then, for $h \in \mathcal{H}$, we have

$$
\mathbb{E}\left[\exp \left(\mathrm{i}(h, X(t))_{\mathcal{H}}\right)\right]=\mathbb{E}\left[\exp \left(\int_{0}^{t} \Psi_{L}\left((\Gamma(t, s)(\sigma(s)))^{*} h\right) d s\right)\right],
$$

where $\Psi_{L}$ is the cumulant functional of $L(1)$.

Proof. Let $\left\{s_{i}\right\}_{i=1}^{n}$ be a partition of $[0, t]$ and denote $\Delta s_{i}=s_{i+1}-s_{i}$ and $\Delta L\left(s_{i}\right)=L\left(s_{i+1}\right)-L\left(s_{i}\right)$ for $i=1, \ldots, n-1$. Then, by the independent increment property of $L$ and double conditioning using the independence between $\sigma$ and $L$, we find

$$
\begin{aligned}
\mathbb{E}\left[\exp \left(\mathrm{i}\left(h, \sum_{i=1}^{n-1} \Gamma\left(t, s_{i}\right)\left(\sigma\left(s_{i}\right)\right) \Delta L\left(s_{i}\right)\right)_{\mathcal{H}}\right)\right] \\
=\mathbb{E}\left[\mathbb{E}\left[\exp \left(\mathrm{i}\left(h, \sum_{i=1}^{n-1} \Gamma\left(t, s_{i}\right)\left(\sigma\left(s_{i}\right)\right) \Delta L\left(s_{i}\right)\right)_{\mathcal{H}}\right) \mid \sigma(\cdot)\right]\right] \\
=\mathbb{E}\left[\prod_{i=1}^{n-1} \mathbb{E}\left[\exp \left(\mathrm{i}\left(h, \Gamma\left(t, s_{i}\right)\left(\sigma\left(s_{i}\right)\right) \Delta L\left(s_{i}\right)\right)_{\mathcal{H}}\right) \mid \sigma(\cdot)\right]\right] \\
=\mathbb{E}\left[\prod_{i=1}^{n-1} \mathbb{E}\left[\exp \left(\mathrm{i}\left(\left(\Gamma\left(t, s_{i}\right)\left(\sigma\left(s_{i}\right)\right)\right)^{*} h, \Delta L\left(s_{i}\right)\right)_{\mathcal{H}}\right) \mid \sigma(\cdot)\right]\right] \\
=\mathbb{E}\left[\prod_{i=1}^{n-1} \exp \left(\Psi_{L}\left(\left(\Gamma\left(t, s_{i}\right)\left(\sigma\left(s_{i}\right)\right)\right)^{*} h\right) \Delta s_{i}\right)\right] .
\end{aligned}
$$

The last equality follows from the Lévy-Kintchine formula for $L$ (see Peszat and Zabczyk [20, Thm. 4.27]). By the Cauchy-Schwarz inequality it holds that

$$
\mathbb{E}\left[\left|(h, X(t))_{\mathcal{H}}-\left(h, X_{n}(t)\right)_{\mathcal{H}}\right|\right] \leq|h|_{\mathcal{H}} \mathbb{E}\left[\left|X(t)-X_{n}(t)\right|_{\mathcal{H}}^{2}\right]^{1 / 2}
$$

where $X_{n}(t)$ is defined by (2.7). Thus, invoking the inequality $\left|\mathrm{e}^{\mathrm{i} x}-\mathrm{e}^{\mathrm{i} y}\right| \leq|x-y|$, for $x, y \in \mathbb{R}$, and Lemma 2.3, complete the proof.

Consider $L=W$, a Wiener process in $\mathcal{H}$. Then, the cumulant functional of $W(1)$ is $\Psi_{W}(v)=$ $-\frac{1}{2}(\mathcal{Q} v, v)_{\mathcal{V}}$ (see Peszat and Zabczyk [20, Thm. 4.27]). If $\sigma$ is independent of $W$, we find by Proposition 2.4 that for any $h \in \mathcal{H}$

$$
\begin{aligned}
\mathbb{E}\left[\exp \left(\mathrm{i}(h, X(t))_{\mathcal{H}}\right)\right] & =\mathbb{E}\left[\exp \left(-\frac{1}{2} \int_{0}^{t}\left(\mathcal{Q}(\Gamma(t, s)(\sigma(s)))^{*} h,(\Gamma(t, s)(\sigma(s)))^{*} h\right) \mathcal{V} d s\right)\right] \\
& =\mathbb{E}\left[\exp \left(-\frac{1}{2} \int_{0}^{t}\left(h, \Gamma(t, s)(\sigma(s)) \mathcal{Q}(\Gamma(t, s)(\sigma(s)))^{*} h\right)_{\mathcal{H}} d s\right)\right]
\end{aligned}
$$




$$
=\mathbb{E}\left[\exp \left(-\frac{1}{2}\left(h, \int_{0}^{t} \Gamma(t, s)(\sigma(s)) \mathcal{Q}(\Gamma(t, s)(\sigma(s)))^{*} d s h\right)_{\mathcal{H}}\right)\right] .
$$

We interpret the $d s$-integral in the last expectation as a Bochner integral in the space of operators. In conclusion, for $L=W$ and $\sigma$ independent of $W$, the Hambit field becomes a Gaussian random variable conditional on $\sigma$. Indeed, $\left.X(t)\right|_{\sigma(\cdot)}$ is an $\mathcal{H}$-valued Gaussian process with covariance operator

$$
\mathcal{Q}_{\left.X(t)\right|_{\sigma(\cdot)}}=\int_{0}^{t} \Gamma(t, s)(\sigma(s)) \mathcal{Q}(\Gamma(t, s)(\sigma(s)))^{*} d s
$$

and mean equal to zero.

We discuss stationarity for the Hambit process. Let $\Gamma(t, s):=\Gamma(t-s)$ for a moment. Choosing a non-random time-independent volatility $\sigma(s):=\sigma \in \mathcal{V}$, we obtain the characteristic functional of the form

$$
\mathbb{E}\left[\exp \left(\mathrm{i}(h, X(t))_{\mathcal{H}}\right)\right]=\exp \left(\int_{0}^{t} \Psi_{L}\left((G(s))^{*} h\right) d s\right),
$$

where $G(s):=\Gamma(s)(\sigma)$. If $s \mapsto \Psi_{L}\left(\left(G(s)^{*} h\right) \in L^{1}\left(\mathbb{R}_{+}\right)\right.$, then we see that the characteristic functional of $X(t)$ has a limit

$$
\lim _{t \rightarrow \infty} \mathbb{E}\left[\exp \left(\mathrm{i}(h, X(t))_{\mathcal{H}}\right)\right]=\exp \left(\int_{0}^{\infty} \Psi_{L}\left((G(s))^{*} h\right) d s\right) .
$$

Assuming $\int_{0}^{\infty}\|G(s)\|_{\text {op }}^{2} d s<\infty$, we can define the $\mathcal{H}$-valued process

$$
X_{\text {stat }}(t)=\int_{-\infty}^{t} G(s) d L(s)
$$

which has characteristic functional

$$
\mathbb{E}\left[\exp \left(\mathrm{i}\left(h, X_{\text {stat }}(t)\right)_{\mathcal{H}}\right)\right]=\exp \left(\int_{0}^{\infty} \Psi_{L}\left((G(s))^{*} h\right) d s\right) .
$$

Hence, $X(t)$, when $t \rightarrow \infty$ is equal in distribution to $X_{\text {stat }}(t)$. The process $X_{\text {stat }}(t)$ is the stationary version of $X(t)$. We remark that ambit fields are often defined to be stationary processes (see Barndorff-Nielsen and Schmiegel [5]). Letting $L=W$ again, we find that the stationary distribution of $X$ is Gaussian in $\mathcal{H}$ with covariance operator

$$
\mathcal{Q}_{X_{\text {stat }}}=\int_{0}^{\infty} G(s) \mathcal{Q} G(s)^{*} d s,
$$

and mean equal to zero. As a specific example of an Hambit field which is asymptotically stationary, we might consider the Ornstein-Uhlenbeck process $(2.3)$ with constant non-random volatility. In this case $\Gamma(t, s)=\mathcal{S}_{t-s}$, where $\mathcal{S}$ is the $C_{0}$-semigroup generated by $\mathcal{A}$.

2.1. Relation to classical ambit fields. We relate Hambit fields to the classical definition of ambit fields, see (1.1).

Let $\mathcal{U}$ be a Hilbert space of real-valued functions on a Borel measurable subset $A \subset \mathbb{R}^{n}, n \in \mathbb{N}$. Consider the measurable real-valued function $(t, s, x, y) \mapsto g(t, s, x, y)$, where $0 \leq s \leq t<\infty, x \in B$, $y \in A$, and $B \subset \mathbb{R}^{d}, d \in \mathbb{N}$ being a Borel measurable subset. Let $\mathcal{V}$ be a Hilbert space of measures on the Borel subsets of $A$. For $\sigma \in \mathcal{U}$, we define the linear operator on $\mathcal{V}$

given by

$$
\Gamma(t, s)(\sigma):=\int_{A} g(t, s, \cdot, y) \sigma(y),
$$

$$
\Gamma(t, s)(\sigma) \mu=\int_{A} g(t, s, \cdot, y) \sigma(y) \mu(d y),
$$

for any $\mu \in \mathcal{V}$. If we let $\mathcal{H}$ be a Hilbert space of real-valued functions on $B$, then under appropriate hypotheses on $g$ and selection of Hilbert spaces one can have $\Gamma(t, s)(\sigma) \mu \in \mathcal{H}$ for $\mu \in \mathcal{V}$ and $\Gamma(t, s) \in$ $L(\mathcal{U}, L(\mathcal{V}, \mathcal{H}))$. Assume $\sigma(s)$ is a $\mathcal{U}$-valued stochastic process such that $\Gamma(t, s)(\sigma(s))$ is integrable with respect to the $\mathcal{V}$-valued Lévy process $L$. Then we get,

$$
X(t, x)=\int_{0}^{t} \int_{A} g(t, s, x, y) \sigma(s, y) L(d y, d s),
$$


which is a classical ambit field. Note that we choose here to work with a kernel function $g$ which is nonstationary in time. In $X(t, x)$ above, the Lévy process $L$ is a measure. A Lévy basis is not a measure, but very close to one (see Barndorff-Nielsen, Benth and Veraart [1] for a discussion of Hilbert-valued processes and Lévy bases).

To be more specific, choose $n=d=1$ and let $A=B=\mathbb{R}_{+}$. Assume $\mathcal{U}=\mathcal{V}=\mathcal{H}$, and let $\mathcal{U}$ be the Filipovic space (see Filipovic [17]) of absolutely continuous functions on $\mathbb{R}_{+}$, that is, real-valued functions $f$ on $\mathbb{R}_{+}$which are weakly differentiable and such that

$$
|f|_{w}^{2}:=f^{2}(0)+\int_{0}^{\infty} w(y)\left|f^{\prime}(y)\right|^{2} d y
$$

for a non-decreasing weight function $w: \mathbb{R}_{+} \rightarrow[1, \infty)$ satisfying $\int_{0}^{\infty} w^{-1}(y) d y<\infty$. We denote this separable Hilbert space $\mathcal{U}_{w}$, and its inner product by $(\cdot, \cdot)_{w}$. For $\sigma \in \mathcal{U}_{w}$, we need to impose conditions on $g$ such that

$$
\Gamma(t, s)(\sigma) f=\int_{0}^{\infty} g(t, s, \cdot, y) \sigma(y) f^{\prime}(y) d y
$$

is an element in $\mathcal{U}_{w}$ for all $(t, s)$ with $s \leq t<\infty$ and $f \in \mathcal{U}_{w}$. Next, we need to have that $\Gamma(t, s)(\sigma) \in$ $L\left(\mathcal{U}_{w}\right)$ and $\Gamma(t, s) \in L\left(\mathcal{U}_{w}, L\left(\mathcal{U}_{w}\right)\right)$, and furthermore that $s \mapsto \Gamma(t, s)(\sigma(s))$ is integrable with respect to the $\mathcal{U}_{w}$-valued Lévy process $L$. We collect the conditions in the next Lemma:

Lemma 2.5. Let $\sigma$ be a predictable $\mathcal{U}_{w}$-valued process, and suppose that $x \mapsto g(t, s, x, y) \in \mathcal{U}_{w}$ for a.e. $(t, s, y)$ is such that

- for a.e. $t \geq s \geq 0$

$$
\int_{0}^{\infty} w^{-1}(y)|g(t, s, \cdot, y)|_{w}^{2} d y<\infty
$$

- and $t \geq 0$,

$$
\int_{0}^{\infty} w^{-1}(y) \int_{0}^{t}|g(t, s, \cdot, y)|_{w}^{2} \mathbb{E}\left[|\sigma(s)|_{w}^{2}\right] d s d y<\infty .
$$

Then we have a classical ambit field

$$
X(t, x)=\int_{0}^{t} \int_{0}^{\infty} g(t, s, x, y) \sigma(y) L(d y, d s)
$$

with $X(t, \cdot) \in \mathcal{U}_{w}$ for $t<\infty$.

Proof. For $\sigma_{1}, \sigma_{2} \in \mathcal{U}_{w}$, we obviously have $\Gamma(t, s)\left(\sigma_{1}+\sigma_{2}\right)=\Gamma(t, s)\left(\sigma_{1}\right)+\Gamma(t, s)\left(\sigma_{2}\right)$. Moreover, for $f_{1}, f_{2} \in \mathcal{U}_{w}$, it is also straightforward to see that $\Gamma(t, s)(\sigma)\left(f_{1}+f_{2}\right)=\Gamma(t, s)(\sigma) f_{1}+\Gamma(t, s)(\sigma) f_{2}$. Thus, to prove that $\Gamma(t, s) \in L\left(\mathcal{U}_{w}, L\left(\mathcal{U}_{w}\right)\right)$ we must show that the linear operators are bounded.

To this end, note that

$$
\|\Gamma(t, s)\|_{\text {op }}=\sup _{|\sigma|_{w} \leq 1}\|\Gamma(t, s)(\sigma)\|_{\text {op }}=\sup _{|\sigma|_{w},|f|_{w} \leq 1}\left|\int_{0}^{\infty} g(t, s, \cdot, y) \sigma(y) f^{\prime}(y) d y\right|_{w} .
$$

By definition

$$
|\Gamma(t, s)(\sigma) f|_{w}^{2}=\left(\int_{0}^{\infty} g(t, s, 0, y) \sigma(y) f^{\prime}(y) d y\right)^{2}+\int_{0}^{\infty} w(x)\left(\int_{0}^{\infty} g_{x}(t, s, x, y) \sigma(y) f^{\prime}(y) d y\right)^{2} d y,
$$

where $g_{x}$ denotes the weak derivative with respect to the third argument of $g$. By the Cauchy-Schwartz inequality, we find for the first term

$$
\begin{aligned}
\left(\int_{0}^{\infty} g(t, s, 0, y) \sigma(y) f^{\prime}(y) d y\right)^{2} & =\left(\int_{0}^{\infty} w^{-1 / 2}(y) g(t, s, 0, y) \sigma(y) w^{1 / 2}(y) f^{\prime}(y) d y\right)^{2} \\
& \leq \int_{0}^{\infty} w^{-1}(y) g^{2}(t, s, 0, y) \sigma^{2}(y) d y \int_{0}^{\infty} w(y)\left|f^{\prime}(y)\right|^{2} d y \\
& \leq \int_{0}^{\infty} w^{-1}(y) g^{2}(t, s, 0, y) \sigma^{2}(y) d y|f|_{w}^{2} .
\end{aligned}
$$


But, by the fundamental theorem of calculus and Cauchy-Schwartz' inequality again,

$$
\begin{aligned}
\sigma^{2}(y) & =\left(\sigma(0)+\int_{0}^{y} \sigma^{\prime}(z) d z\right)^{2} \\
& \leq 2 \sigma^{2}(0)+2\left(\int_{0}^{y} \sigma^{\prime}(z) d z\right)^{2} \\
& =2 \sigma^{2}(0)+2\left(\int_{0}^{y} w^{-1 / 2}(z) w^{1 / 2}(z) \sigma^{\prime}(z) d z\right)^{2} \\
& \leq 2 \sigma^{2}(0)+2 \int_{0}^{y} w^{-1}(z) d z \int_{0}^{y} w(z)\left|\sigma^{\prime}(z)\right|^{2} d z \\
& \leq 2\left(1+\int_{0}^{\infty} w^{-1}(z) d z\right)|\sigma|_{w}^{2} .
\end{aligned}
$$

Hence, we find

$$
\left(\int_{0}^{\infty} g(t, s, 0, y) \sigma(y) f^{\prime}(y) d y\right)^{2} \leq 2 \int_{0}^{\infty} w^{-1}(y) g^{2}(t, s, 0, y) d y\left(1+\int_{0}^{\infty} w^{-1}(y) d y\right)|\sigma|_{w}^{2}|f|_{w}^{2} .
$$

For the second integral in $(2.12)$, it follows by similar arguments that,

$$
\begin{aligned}
\int_{0}^{\infty} w(x) & \left(\int_{0}^{\infty} g_{x}(t, s, x, y) \sigma(y) f^{\prime}(y) d y\right)^{2} d y \\
& =\int_{0}^{\infty} w(x)\left(\int_{0}^{\infty} w^{-1 / 2}(y) g_{x}(t, s, x, y) \sigma(y) w^{1 / 2}(y) f^{\prime}(y) d y\right)^{2} d x \\
& \leq \int_{0}^{\infty} w(x) \int_{0}^{\infty} w^{-1}(y) g_{x}^{2}(t, s, x, y) \sigma^{2}(y) d y d x|f|_{w}^{2} \\
& =\int_{0}^{\infty} \int_{0}^{\infty} w(x) w^{-1}(y) g_{x}^{2}(t, s, x, y) d x \sigma^{2}(y) d y|f|_{w}^{2} \\
& \leq 2 \int_{0}^{\infty} w^{-1}(y)\left(\int_{0}^{\infty} w(x) g_{x}^{2}(t, s, x, y) d x\right) d y\left(1+\int_{0}^{\infty} w^{-1}(z) d z\right)|\sigma|_{w}^{2}|f|_{w}^{2}
\end{aligned}
$$

These estimations imply

$$
\|\Gamma(t, s)(\sigma)\|_{\mathrm{op}}^{2} \leq|\sigma|_{w}^{2} 2\left(1+\int_{0}^{\infty} w^{-1}(z) d z\right) \int_{0}^{\infty} w^{-1}(y)|g(t, s, \cdot, y)|_{w}^{2} d y,
$$

and

$$
\|\Gamma(t, s)\|_{\text {op }}^{2} \leq 2\left(1+\int_{0}^{\infty} w^{-1}(z) d z\right) \int_{0}^{\infty} w^{-1}(y)|g(t, s, \cdot, y)|_{w}^{2} d y,
$$

and therefore $\Gamma \in L\left(\mathcal{U}_{w}, L\left(\mathcal{U}_{w}\right)\right)$ by the assumptions of the Lemma.

For the $L$-integrability, we first note that since $\sigma(s)$ is assumed to be predictable, it follows that $s \mapsto$ $\Gamma(s, t)(\sigma(s))$ is predictable. We must show that the integrability condition (2.1) holds:

$$
\begin{aligned}
\mathbb{E}\left[\int_{0}^{t}\left\|\Gamma(t, s)(\sigma(s)) \mathcal{Q}^{1 / 2}\right\|_{\mathrm{HS}}^{2} d s\right] & \leq \mathbb{E}\left[\int_{0}^{t}\|\Gamma(t, s)(\sigma(s))\|_{\mathrm{op}}^{2}\left\|\mathcal{Q}^{1 / 2}\right\|_{\mathrm{HS}}^{2} d s\right] \\
\leq 2\left\|\mathcal{Q}^{1 / 2}\right\|_{\mathrm{HS}}^{2}\left(1+\int_{0}^{\infty} w^{-1}(z) d z\right) & \times \int_{0}^{\infty} w^{-1}(y) \int_{0}^{t}|g(t, s, \cdot, y)|_{w}^{2} \mathbb{E}\left[|\sigma(s)|_{w}^{2}\right] d s d y
\end{aligned}
$$

which is finite by the assumptions of the Lemma. Hence, the proof is complete.

In the representation of $X(t, x)$, we have used the notation $L(d y, d s)=\partial_{y} L(y, d s) d y$, where $\partial_{y}$ is the partial (weak) derivative with respect to $y$. We remark that we can define the classical ambit field

$$
X(t, x)=\int_{0}^{t} \int_{A} g(t, s, x, y) \sigma(s) L(d y, d s)
$$


by

$$
X(t, x)=\int_{0}^{t} \int_{0}^{\infty} g(t, s, x, y) 1(y \in A) \sigma(s) L(d y, d s),
$$

for some Borel measurable subset $A \subset \mathbb{R}_{+}$. We note that

$$
|g(t, s, \cdot, y) 1(y \in A)|_{w}=1(y \in A)|g(t, s, \cdot, y)|_{w},
$$

and therefore

$$
\int_{0}^{\infty} w^{-1}(y)|g(t, s, \cdot, y) 1(y \in A)|_{w}^{2} d y=\int_{A} w^{-1}(y)|g(t, s, \cdot, y)|_{w}^{2} d y \leq \int_{0}^{\infty} w^{-1}(y)|g(t, s, \cdot, y)|_{w}^{2} d y .
$$

Thus, either we can impose slightly weaker $\mathcal{U}_{w}$-norm-integrability conditions on $g$ (the middle estimate above), or we can assume the strong one given in Lemma 2.5. In the latter case, we observe that such a condition provides us with a classical ambit field for all choices of $A$.

2.2. Representation of Hambit fields in terms of volatility modulated Volterra processes. We show that a Hambit field can be represented as a countable sum of volatility modulated Volterra (VMV) processes under a certain regularity condition on the stochastic volatility field $\sigma$ :

Proposition 2.6. Let $\left\{u_{n}\right\}_{n \in \mathbb{N}},\left\{v_{m}\right\}_{m \in \mathbb{N}}$ and $\left\{h_{k}\right\}_{k \in \mathbb{N}}$ be ONB's in $\mathcal{U}, \mathcal{V}$ and $\mathcal{H}$, resp. Suppose that

$$
\int_{0}^{t}\|\Gamma(t, s)\|_{o p}^{2}\left(\sum_{n=1}^{\infty} \mathbb{E}\left[\left(\sigma(s), u_{n}\right)_{\mathcal{U}}^{2}\right]^{1 / 2}\right)^{2} d s<\infty .
$$

Then the Hambit field $X(t)$ can be represented in $L^{2}(\Omega)$ as

$$
X(t)=\sum_{n, m, k=1}^{\infty} Y_{n, m, k}(t) h_{k},
$$

where $t \mapsto Y_{n, m, k}(t), 0 \leq t \leq T, n, m, k \in \mathbb{N}$ are real-valued $V M V$ processes defined by

$$
Y_{n, m, k}(t)=\int_{0}^{t}\left(\Gamma(t, s)\left(u_{n}\right) v_{m}, h_{k}\right)_{\mathcal{H}}\left(\sigma(s), u_{n}\right) \mathcal{U} d L_{m}(s),
$$

and $L_{m}:=\left(L, v_{m}\right)_{\mathcal{V}}, m \in \mathbb{N}$ are real-valued square integrable Lévy processes with zero mean.

Proof. We can represent $L(t)$ by

$$
L(t)=\sum_{m=1}^{\infty}\left(L(t), v_{m}\right) \mathcal{v} v_{m}
$$

where $L_{m}:=\left(L, v_{m}\right) \mathcal{V}$ is a real-valued square integrable mean zero Lévy process. Hence,

$$
X(t)=\sum_{m=1}^{\infty} \int_{0}^{t} \Gamma(t, s)(\sigma(s)) v_{m} d L_{m}(s) .
$$

$\operatorname{But} \Gamma(t, s)(\sigma(s)) v_{m} \in \mathcal{H}$, and thus the stochastic integral $\int_{0}^{t} \Gamma(t, s)(\sigma(s)) v_{m} d L_{m}(s) \in \mathcal{H}$ as well. Hence, a.s.,

$$
\begin{aligned}
\int_{0}^{t} \Gamma(t, s)(\sigma(s)) v_{m} d L_{m}(s) & =\sum_{k=1}^{\infty}\left(\int_{0}^{t} \Gamma(t, s)(\sigma(s)) v_{m} d L_{m}(s), h_{k}\right)_{\mathcal{H}} h_{k} \\
& =\sum_{k=1}^{\infty} \int_{0}^{t}\left(\Gamma(t, s)(\sigma(s)) v_{m}, h_{k}\right)_{\mathcal{H}} d L_{m}(s) h_{k} .
\end{aligned}
$$

The last equality follows by definition of the stochastic integral of an $\mathcal{H}$-valued adapted process with respect to a real-valued Lévy process. This means that

$$
X(t)=\sum_{m, k=1}^{\infty} \int_{0}^{t}\left(\Gamma(t, s)(\sigma(s)) v_{m}, h_{k}\right)_{\mathcal{H}} d L_{m}(s) h_{k} .
$$


Finally, express $\sigma(s)=\sum_{n=1}^{\infty}\left(\sigma(s), u_{n}\right) \mathcal{U} u_{n}$ to find

$$
\left(\Gamma(t, s)(\sigma(s)) v_{m}, h_{k}\right)_{\mathcal{H}}=\sum_{n=1}^{\infty}\left(\sigma(s), u_{n}\right) \mathcal{U}\left(\Gamma(t, s)\left(u_{n}\right) v_{m}, h_{k}\right)_{\mathcal{H}}
$$

by linearity of the inner product and continuity of the operator $\Gamma(t, s)$. We show next that

$$
\int_{0}^{t}\left(\Gamma(t, s)(\sigma(s)) v_{m}, h_{k}\right)_{\mathcal{H}} d L_{m}(s)=\sum_{n=1}^{\infty} \int_{0}^{t}\left(\Gamma(t, s)\left(u_{n}\right) v_{m}, h_{k}\right)_{\mathcal{H}}\left(\sigma(s), u_{n}\right) \mathcal{U} d L_{m}(s) .
$$

Note that, as the Lévy process $L_{m}$ is a square-integrable martingale, we find by the definition of stochastic integration with respect to martingales (see e.g. Protter [21])

$$
\begin{gathered}
\mathbb{E}\left[\left(\int_{0}^{t}\left(\Gamma(t, s)(\sigma(s)) v_{m}, h_{k}\right)_{\mathcal{H}} d L_{m}(s)-\sum_{n=1}^{N} \int_{0}^{t}\left(\Gamma(t, s)\left(u_{n}\right) v_{m}, h_{k}\right) \mathcal{H}\left(\sigma(s), u_{n}\right) \mathcal{U} d L_{m}(s)\right)^{2}\right] \\
=\mathbb{E}\left[\left(\int_{0}^{t} \sum_{n=N+1}^{\infty}\left(\Gamma(t, s)\left(u_{n}\right) v_{m}, h_{k}\right)_{\mathcal{H}}\left(\sigma(s), u_{n}\right) \mathcal{U} d L_{m}(s)\right)^{2}\right] \\
=\mathbb{E}\left[L_{m}^{2}(1)\right] \int_{0}^{t} \mathbb{E}\left[\left(\sum_{n=N+1}^{\infty}\left(\Gamma(t, s)\left(u_{n}\right) v_{m}, h_{k}\right)_{\mathcal{H}}\left(\sigma(s), u_{n}\right) \mathcal{U}\right)^{2}\right] d s
\end{gathered}
$$

By Minkowski’s inequality (see Folland [18, p. 186]), we have

$$
\begin{gathered}
\mathbb{E}\left[\left(\sum_{n=N+1}^{\infty}\left(\Gamma(t, s)\left(u_{n}\right) v_{m}, h_{k}\right)_{\mathcal{H}}\left(\sigma(s), u_{n}\right) \mathcal{U}\right)^{2}\right]^{1 / 2} \\
\leq \sum_{n=N+1}^{\infty} \mathbb{E}\left[\left(\Gamma(t, s)\left(u_{n}\right) v_{m}, h_{k}\right)_{\mathcal{H}}^{2}\left(\sigma(s), u_{n}\right)_{\mathcal{U}}^{2}\right]^{1 / 2} \\
\leq\|\Gamma(t, s)\|_{\text {op }} \sum_{n=N+1}^{\infty} \mathbb{E}\left[\left(\sigma(s), u_{n}\right)_{\mathcal{U}}^{2}\right]^{1 / 2}
\end{gathered}
$$

since, using that the bases are orthonormal,

$$
\left.\left|\left(\Gamma(t, s)\left(u_{n}\right) v_{m}, h_{k}\right) \mathcal{H}\right|^{2} \leq \mid \Gamma(t, s)\left(u_{n}\right) v_{m}\right)\left.\right|_{\mathcal{H}} ^{2}\left|h_{k}\right|_{\mathcal{H}}^{2} \leq\left\|\Gamma(t, s)\left(u_{n}\right)\right\|_{\text {op }}^{2}\left|v_{m}\right|_{\mathcal{V}}^{2} \leq\|\Gamma(t, s)\|_{\text {op }}^{2}\left|u_{n}\right|_{\mathcal{U}}^{2} .
$$

Hence,

$$
\begin{gathered}
\int_{0}^{t} \mathbb{E}\left[\left(\sum_{n=N+1}^{\infty}\left(\Gamma(t, s)\left(u_{n}\right) v_{m}, h_{k}\right)_{\mathcal{H}}\left(\sigma(s), u_{n}\right) \mathcal{U}\right)^{2}\right] d s \\
\leq \int_{0}^{t}\|\Gamma(t, s)\|_{\text {op }}^{2}\left(\sum_{n=N+1}^{\infty} \mathbb{E}\left[\left(\sigma(s), u_{n}\right)_{\mathcal{U}}^{2}\right]^{1 / 2}\right)^{2} d s
\end{gathered}
$$

which tends to zero as $N \rightarrow \infty$ by assumption. The result follows.

Remark that the real-valued Lévy processes $\left\{L_{m}\right\}_{m=1}^{\infty}$ defined in Prop. 2.6 above are not independent. They are not even zero correlated unless the ONB $\left\{v_{m}\right\}_{k \in \mathbb{N}}$ consists of the eigenvectors of $\mathcal{Q}$. Indeed, we have

$$
\mathbb{E}\left[\left(L(t), v_{m}\right) \mathcal{V}\left(L(t), v_{k}\right) \mathcal{V}\right]=\left(\mathcal{Q} v_{m}, v_{k}\right) \mathcal{V},
$$

for $k, m \in \mathbb{N}$. Further, we also observe that if $\Gamma(t, s)=\Gamma(t-s)$, i.e., the kernel is specified in a stationary form, then the real-valued processes $Y_{n, m, k}(t)$ in Prop.2.6 become,

$$
Y_{n, m, k}(t)=\int_{0}^{t}\left(\Gamma(t-s)\left(u_{n}\right) v_{m}, h_{k}\right)_{\mathcal{H}}\left(\sigma(s), u_{n}\right) \mathcal{U} d L_{m}(s),
$$


which is in fact a Lévy semistationary (LSS) process. Barndorff-Nielsen, Benth and Veraart [2] applied LSS processes to model spot prices in energy market. Further, using factor models involving Lévy-driven continuous-time autoregressive moving average processes to describe electricity spot prices, Benth et al. [10] extended the classical commodity spot market models based on Wiener-driven Ornstein-Uhlenbeck processes. The class of continuous-time autoregressive moving average processes is a special case of LSS processes (see Brockwell [13], and Benth and Šaltytė Benth [7] for an analysis and discussion in weather modelling). Barndorff-Nielsen, Benth and Veraart [3] proposed ambit fields as a modeling tool for energy forward markets. Our result in Prop.2.6 shows that any ambit field can be represented as an infinite LSS (or VMV) factor model, providing a strong theoretical argument for the rationale in using LSS (or VMV) processes and ambit fields as modelling devices for commodity market prices.

The integrability condition on $\Gamma$ and $\sigma$ in Prop.2.6 is stronger than the sufficient condition in Lemma2.2 for well-definedness of the Hambit field $X$. In fact, by Parseval's identity (and Tonelli's theorem)

$$
\mathbb{E}\left[|\sigma(s)|_{\mathcal{U}}^{2}\right]=\sum_{n=1}^{\infty} \mathbb{E}\left[\left(\sigma(s), u_{n}\right)_{\mathcal{U}}^{2}\right]
$$

for $\left\{u_{n}\right\}_{n \in \mathbb{N}}$ ONB of $\mathcal{U}$. As long as $\sum_{n=1}^{\infty} \mathbb{E}\left[\left(\sigma(s), u_{n}\right)_{\mathcal{U}}^{2}\right]^{1 / 2}<\infty$, there exists $N \in \mathbb{N}$ such that $\mathbb{E}\left[\left(\sigma(s), u_{n}\right)_{\mathcal{U}}^{2}\right]^{1 / 2}<1$ for $n \geq N$. Hence $\mathbb{E}\left[\left(\sigma(s), u_{n}\right)_{\mathcal{U}}^{2}\right] \leq \mathbb{E}\left[\left(\sigma(s), u_{n}\right)_{\mathcal{U}}^{2}\right]^{1 / 2}$. Thus, the condition in Prop. 2.6 implies that the condition of Lemma 2.2 holds. Suppose now that $\left\{a_{n}\right\}_{n \in \mathbb{N}}$ is a sequence of strictly positive numbers such that $\sum_{n=1}^{\infty} a_{n}^{-1}<\infty$. Then, by the Cauchy-Schwarz inequality

$$
\left(\sum_{n=1}^{\infty} \mathbb{E}\left[\left(\sigma(s), u_{n}\right)_{\mathcal{U}}^{2}\right]^{1 / 2}\right)^{2}=\left(\sum_{n=1}^{\infty} a_{n}^{-1}\right)\left(\sum_{n=1}^{\infty} a_{n} \mathbb{E}\left[\left(\sigma(s), u_{n}\right)_{\mathcal{U}}^{2}\right]\right)
$$

Thus,

$$
\sum_{n=1}^{\infty} a_{n} \int_{0}^{t}\|\Gamma(t, s)\|_{\mathrm{op}}^{2} \mathbb{E}\left[\left(\sigma(s), u_{n}\right)_{\mathcal{U}}^{2}\right] d s<\infty
$$

is a sufficient condition for Prop. 2.6 to hold.

Let us consider an example. Let $U$ be a $\mathcal{U}$-valued square-integrable Lévy process with zero mean and $\eta \in L^{2}\left(\mathbb{R}_{+}\right)$. Assume that $\sigma(t)$ is the $\mathcal{U}$-valued OU process

$$
\sigma(t)=\int_{0}^{t} \eta(t-s) d U(s) .
$$

This is a very simple definition of an LSS-process with values in the Hilbert space $\mathcal{U}$. As $U$ is squareintegrable, it has a covariance operator $\mathcal{Q}_{U}$ on $\mathcal{U}$, and we assume that the ONB $\left\{u_{n}\right\}$ is the set of eigenvectors of $\mathcal{Q}_{U}$ with corresponding eigenvalues $\lambda_{n}$. As $\mathcal{Q}_{U}$ is positive definite, we have $0 \leq\left(\mathcal{Q}_{U} u_{n}, u_{n}\right) \mathcal{U}=$ $\lambda_{n}$, i.e., all eigenvalues are non-negative. We find that

$\left(\sigma(t), u_{n}\right) \mathcal{U}=\sum_{k=1}^{\infty} \int_{0}^{t}\left(\eta(t-s) u_{k}, u_{n}\right) \mathcal{U} d U_{k}(s)=\sum_{k=1}^{\infty} \int_{0}^{t} \eta(t-s)\left(u_{k}, u_{n}\right) \mathcal{U} d U_{k}(s)=\int_{0}^{t} \eta(t-s) d U_{n}(s)$

where $U_{n}(s)=\left(U(s), u_{n}\right)_{\mathcal{U}}$ is a square integrable real-valued Lévy process with zero mean. But then,

$$
\mathbb{E}\left[\left(\sigma(t), u_{n}\right)_{\mathcal{U}}^{2}\right]=\mathbb{E}\left[\left(\int_{0}^{t} \eta(t-s) d U_{n}(s)\right)^{2}\right]=\lambda_{n} \int_{0}^{t} \eta^{2}(s) d s .
$$

The integrability condition in Prop. 2.6 thus becomes

$$
\int_{0}^{t}\|\Gamma(t, s)\|_{\mathrm{op}}^{2}\left(\sum_{n=1}^{\infty} \sqrt{\lambda_{n}}\left(\int_{0}^{s} \eta^{2}(v) d v\right)^{1 / 2}\right)^{2} d s=\left(\sum_{n=1}^{\infty} \sqrt{\lambda_{n}}\right)^{2} \int_{0}^{t}\|\Gamma(t, s)\|_{\mathrm{op}}^{2} \int_{0}^{s} \eta^{2}(v) d v d s .
$$

Hence, since $\eta \in L^{2}\left(\mathbb{R}_{+}\right)$, the integrability condition in Prop.2.6 is satisfied if $\int_{0}^{t}\|\Gamma(t, s)\|_{\mathrm{op}}^{2} d s<\infty$ and $\sum_{n=1}^{\infty} \sqrt{\lambda_{n}}<\infty$. Note that $\infty>\left\|\mathcal{Q}_{\mathcal{U}}\right\|_{\mathrm{HS}}^{2}=\sum_{n=1}^{\infty} \lambda_{n}$, which is weaker than the summability of $\sqrt{\lambda_{n}}$. We find that $\operatorname{Tr}\left(\mathcal{Q}_{\mathcal{U}}^{1 / 2}\right)=\sum_{n=1}^{\infty} \sqrt{\lambda_{n}}$, so the summability of $\sqrt{\lambda_{n}}$ is equivalent to assuming that $\mathcal{Q}_{\mathcal{U}}^{1 / 2}$ has finite trace. 
A natural application of Prop. 2.6 is to truncate the infinite sum in order to obtain an approximation of the Hambit field $X$. For this purpose, define

$$
X_{N, M, K}(t):=\sum_{n=1}^{N} \sum_{m=1}^{M} \sum_{k=1}^{K} Y_{n, m, k}(t) h_{k}
$$

for $N, M, K \geq 1$, where $Y_{n, m, k}(t)$ is given in Proposition 2.6. It moreover follows by a repeated application of Minkowski's inequality (see Folland [18, p. 186]) that

$$
\left(\mathbb{E}\left[\left|X(t)-X_{N, M, K}(t)\right|_{\mathcal{H}}^{2}\right]\right)^{1 / 2} \leq \sum_{n=N+1}^{\infty} \sum_{m=M+1}^{\infty} \sum_{k=K+1}^{\infty}\left|h_{k}\right|_{\mathcal{H}}\left(\mathbb{E}\left[\left|Y_{n, m, k}(t)\right|^{2}\right]\right)^{1 / 2},
$$

and note furthermore that

$$
\mathbb{E}\left[\left|Y_{n, m, k}(t)\right|^{2}\right] \leq\left(\mathbb{E}\left[\left|L_{m}(1)\right|^{2}\right]\right)^{1 / 2} \int_{0}^{t}\left|\left(\Gamma(t, s)\left(u_{n}\right) v_{m}, h_{k}\right) \mathcal{H}\right|^{2} \mathbb{E}\left[\left|\left(\sigma(s), u_{n}\right) \mathcal{u}\right|^{2}\right] d s .
$$

Given the respective ONB's, one can thus make the error induced by means of the truncated Hambit field (2.14) arbitrarily small. The rate of convergence, on the other hand, is not easily derived in the general set-up, and requires some more structure on the Hilbert spaces to be quantified.

Sometimes it may be convenient to express the Hambit field in terms of a finite set of given "nice" vectors in $\mathcal{H}$. To this end, let $\left\{\xi_{1}, \xi_{2}, \ldots, \xi_{n}\right\}$ be $n$ linearly independent elements of $\mathcal{H}$, and denote by $\mathcal{H}_{n}$ the subspace of $\mathcal{H}$ spanned by these. Note that $\left\{\xi_{i}\right\}_{i=1}^{n}$ may be a subset of the basis functions of $\mathcal{H}$, but in general they are not. Introduce the projection operator $\mathcal{P}_{n}: \mathcal{H} \rightarrow \mathcal{H}_{n}$ defined as

$$
\mathcal{P}_{n}(f)=\left(f, \vec{\xi}_{n}\right)_{\mathcal{H}}^{\prime} H_{n}^{-1} \vec{\xi}_{n},
$$

for $f \in \mathcal{H}$. Here, $\left(f, \vec{\xi}_{n}\right)_{\mathcal{H}}^{\prime}=\left(\left(f, \xi_{1}\right)_{\mathcal{H}}, \ldots,\left(f, \xi_{n}\right)_{\mathcal{H}}\right)^{\prime} \in \mathbb{R}^{n}, \vec{\xi}_{n}$ is the vector with coordinates $\xi_{1}, \xi_{2}, \ldots, \xi_{n}$, and $H_{n}$ the symmetric $n \times n$-matrix with coordinates $\left(\xi_{i}, \xi_{j}\right)_{\mathcal{H}}, i, j=1, \ldots, n$ assumed to be invertible. We recall from basic functional analysis that $\mathcal{P}_{n}(f)$ is the element in $\mathcal{H}_{n}$ which minimizes the distance, that is, $\left|f-\mathcal{P}_{n}(f)\right|_{\mathcal{H}}=\inf _{g \in \mathcal{H}_{n}}|f-g|_{\mathcal{H}}$. In the next Proposition we state the representation of an Hambit field projected down on $\mathcal{H}_{n}$ in the Gaussian case:

Proposition 2.7. Let $L=W$ be an $\mathcal{V}$-valued Wiener process. Then for any $n \in \mathbb{N}$ there exists an $n$ dimensional standard Brownian motion $\vec{B}(t)=\left(B_{1}(t), \ldots, B_{n}(t)\right)$ such that

$$
\mathcal{P}_{n}(X(t))=\int_{0}^{t} \gamma(t, s) d \vec{B}(s)^{\prime} H_{n}^{-1} \vec{\xi}_{n},
$$

with $\gamma(t, s)$ being the square-root of the symmetric, positive definite stochastic $n \times n$ variance-covariance matrix

$$
C(t, s)=\left\{\left(\mathcal{Q}^{1 / 2} \Gamma(t, s)(\sigma(s))^{*} \xi_{i}, \mathcal{Q}^{1 / 2} \Gamma(t, s)(\sigma(s))^{*} \xi_{j}\right) \mathcal{V}\right\}_{i, j=1}^{n} .
$$

Proof. By definition, we have

$$
\mathcal{P}_{n}(X(t))=\left(X(t), \vec{\xi}_{n}\right)_{\mathcal{H}}^{\prime} H_{n}^{-1} \vec{\xi}_{n}
$$

which can be written as

$$
\mathcal{P}_{n}(X(t))=\mathcal{T}_{n}(X(t))^{\prime} H_{n}^{-1} \vec{\xi}_{n},
$$

for the operator $\mathcal{T}_{n} \in L\left(\mathcal{H}, \mathbb{R}^{n}\right)$ defined by

$$
\mathcal{T}_{n}(f)=\left(\left(f, h_{1}\right)_{\mathcal{H}}, \ldots,\left(f, h_{n}\right)_{\mathcal{H}}\right)^{\prime} .
$$

Note that for any $\vec{x} \in \mathbb{R}^{n}$, we have

$$
\mathcal{T}_{n}(f)^{\prime} \vec{x}=\left(f, \mathcal{T}_{n}^{*}(\vec{x})\right)_{\mathcal{H}},
$$

and therefore $\mathcal{T}_{n}^{*} \in L\left(\mathbb{R}^{n}, \mathcal{H}\right)$ is

$$
\mathcal{T}_{n}^{*}(\vec{x})=\vec{x}^{\prime} \vec{\xi}_{n} .
$$

From Thm. 2.1 in Benth and Krühner [11], we obtain the existence of an $n$-dimensional Brownian motion $\vec{B}$ such that

$$
\mathcal{T}_{n}(X(t))=\int_{0}^{t} \gamma(t, s) d \vec{B}(s)
$$


for $\gamma(t, s) \in \mathbb{R}^{n \times n}$ where

$$
\gamma(t, s)^{2}=\mathcal{T}_{n} \Gamma(t, s)(\sigma(s)) \mathcal{Q} \Gamma(t, s)(\sigma(s))^{*} \mathcal{T}_{n}^{*} .
$$

But by definition of the involved operators

$$
\mathcal{T}_{n} \Gamma(t, s)(\sigma(s)) \mathcal{Q} \Gamma(t, s)(\sigma(s))^{*} \mathcal{T}_{n}^{*}(\vec{x})=C(s, t) \vec{x} .
$$

The matrix $C(t, s)$ is obviously symmetric by definition. Since, for any $\vec{x} \in \mathbb{R}^{n}$, we find

$$
\vec{x}^{\mathrm{T}} C(s, t) \vec{x}=\left|\mathcal{Q}^{1 / 2} \Gamma(s, t)(\sigma(s))^{*} h\right|_{\mathcal{V}}^{2} \geq 0,
$$

with $h:=\sum_{i=1}^{n} x_{i} h_{i} \in \mathcal{H}$, positive definiteness of $C(s, t)$ follows. Thus, $C$ has a square-root and the proof is complete.

In a practical situation one aims at choosing $\xi_{i}$ such that the elements in $C$ are easy to compute. We note that $\vec{\beta}_{n}:=H_{n}^{-1 / 2} \vec{\xi}_{n}$ is an $n$-dimensional vector of orthonormal basis elements of $\mathcal{H}_{n}$.

\section{HAMBIT FIELDS AND HYPERBOLIC SPDES}

By a simple change of variables, one may view an Hambit field as the solution of a linear hyperbolic SPDE evaluated at the boundary. In the present Section we analyse this connection in further detail.

To this end, let $\widetilde{\mathcal{H}}$ be a separable Hilbert space of strongly measurable $\mathcal{H}$-valued functions on $\mathbb{R}_{+}$, and denote by $\mathcal{S}_{\xi}$ for $\xi \geq 0$ the right-shift operator defined by $\mathcal{S}_{\xi} f=f(\xi+\cdot)$ for $f \in \widetilde{\mathcal{V}}$. We assume that $\left\{\mathcal{S}_{\xi}\right\}_{\xi \geq 0}$ is a $C_{0}$-semigroup on $\widetilde{\mathcal{H}}$. The generator of $\mathcal{S}_{\xi}$ is seen to be $\partial_{\xi}=\partial / \partial \xi$, being a densely defined unbounded operator on $\widetilde{\mathcal{H}}$.

Consider the SPDE

$$
d Y(t)=\partial_{\xi} Y(t) d t+\Gamma(t+\cdot, t)(\sigma(t)) d L(t),
$$

with initial value $Y(0) \in \widetilde{\mathcal{H}}$. We suppose that for every $f \in \mathcal{H}$ and $t \in \mathbb{R}_{+}$, the mapping

$$
\mathbb{R}_{+} \mapsto \mathcal{H}: \quad \xi \mapsto \Gamma(t+\xi, t)(\sigma(t)) f
$$

is an element of $\widetilde{\mathcal{H}}$ and that $\Gamma(t+\cdot, t)(\sigma(t)) \in L(\mathcal{V}, \widetilde{\mathcal{H}})$. Furthermore, we suppose that

$$
\mathbb{E}\left[\int_{0}^{t}\left\|\Gamma(s+\cdot, s)(\sigma(s)) \mathcal{Q}^{1 / 2}\right\|_{\mathrm{HS}}^{2} d s\right]<\infty
$$

which makes the stochastic integral term in (3.1) well-defined. Remark that the Hilbert-Schmidt norm is with respect to linear operators from $\mathcal{V}$ to $\widetilde{\mathcal{H}}$ and that predictability of the integrand is ensured from the definition of a Hambit field.

Assume the additional integrability condition on the noise term of the SPDE in (3.1),

$$
\mathbb{E}\left[\int_{0}^{t}\left\|\Gamma(t+\cdot, s)(\sigma(s)) \mathcal{Q}^{1 / 2}\right\|_{\mathrm{HS}}^{2} d s\right]<\infty .
$$

Then, by Peszat and Zabczyk [20, Ch. 9] there exists a unique mild solution of (3.1) given by the predictable $\widetilde{\mathcal{H}}$-valued stochastic process $Y(t)$,

$$
\begin{aligned}
Y(t) & =\mathcal{S}_{t} Y(0)+\int_{0}^{t} \mathcal{S}_{t-s} \Gamma(s+\cdot, s)(\sigma(s)) d L(s) \\
& =\mathcal{S}_{t} Y(0)+\int_{0}^{t} \Gamma(t+\cdot, s)(\sigma(s)) d L(s) .
\end{aligned}
$$

We have the following result, which can be used to link $Y$ to the Hambit field $X$.

Proposition 3.1. Suppose $\{\Phi(s)\}_{s \in \mathbb{R}_{+}}$is a predictable process with values in $L(\mathcal{V}, \widetilde{\mathcal{H}})$ being L-integrable.If $\mathcal{L} \in L(\widetilde{\mathcal{H}}, \mathcal{H})$, then

$$
\mathcal{L} \int_{0}^{t} \Phi(s) d L(s)=\int_{0}^{t} \mathcal{L} \Phi(s) d L(s) .
$$


Proof. Note first that $\int_{0}^{t} \Phi(s) d L(s)$ takes values in $\widetilde{\mathcal{H}}$, while $\int_{0}^{t} \mathcal{L} \Phi(s) d L(s)$ takes values in $\mathcal{H}$ since $\mathcal{L} \Phi(s) \in L(\mathcal{V}, \mathcal{H})$. Moreover, since $\mathcal{L}$ is a bounded operator,

$$
|\mathcal{L} \Phi(s) v|_{\mathcal{H}}^{2} \leq\|\mathcal{L}\|_{\text {op }}^{2}|\Phi(s) v|_{\widetilde{\mathcal{H}}}^{2},
$$

and

$$
\mathbb{E}\left[\int_{0}^{t}\left\|\mathcal{L} \Phi(s) \mathcal{Q}^{1 / 2}\right\|_{\mathrm{HS}}^{2} d s\right] \leq\|\mathcal{L}\|_{\mathrm{op}}^{2} \mathbb{E}\left[\int_{0}^{t}\left\|\Phi(s) \mathcal{Q}^{1 / 2}\right\|_{\mathrm{HS}}^{2} d s\right]<\infty
$$

by the integrability assumption on $\Phi$. Thus, $s \mapsto \mathcal{L} \Phi(s)$ is $L$-integrable.

Let $\widetilde{\Phi}$ be a simple process in $L(\mathcal{V}, \widetilde{\mathcal{H}})$, e.g.,

$$
\widetilde{\Phi}(s)=\sum_{k=1}^{n} \widetilde{\Phi}_{k} 1\left(s_{k-1} \leq s<s_{k}\right) .
$$

Then

$$
\mathcal{L} \widetilde{\Phi}(s)=\sum_{k=1}^{n} \mathcal{L} \widetilde{\Phi}_{k} 1\left(s_{k-1} \leq s<s_{k}\right),
$$

is a simple process in $L(\mathcal{V}, \mathcal{H})$, and

$$
\mathcal{L} \int_{0}^{t} \widetilde{\Phi}(s) d L(s)=\mathcal{L} \sum_{k=1}^{n} \widetilde{\Phi}_{k}\left(\Delta L\left(s_{k}\right)\right)=\sum_{k=1}^{n} \mathcal{L} \widetilde{\Phi}_{k}\left(\Delta L\left(s_{k}\right)\right)=\int_{0}^{t} \mathcal{L} \widetilde{\Phi}(s) d L(s) .
$$

Thus, the proposition holds for simple processes.

Let $\left\{\widetilde{\Phi}_{n}\right\}_{n \in \mathbb{N}}$ be a sequence of simple processes such that

$$
\mathbb{E}\left[\int_{0}^{t}\left\|\left(\widetilde{\Phi}_{n}(s)-\Phi(s)\right) \mathcal{Q}^{1 / 2}\right\|_{\mathrm{HS}}^{2} d s\right] \rightarrow 0
$$

when $n \rightarrow \infty$. It holds,

$$
\mathbb{E}\left[\int_{0}^{t}\left\|\left(\mathcal{L} \widetilde{\Phi}_{n}(s)-\mathcal{L} \Phi(s)\right) \mathcal{Q}^{1 / 2}\right\|_{\mathrm{HS}}^{2} d s\right] \leq\|\mathcal{L}\|_{\mathrm{op}}^{2} \mathbb{E}\left[\int_{0}^{t}\left\|\left(\widetilde{\Phi}_{n}(s)-\Phi(s)\right) \mathcal{Q}^{1 / 2}\right\|_{\mathrm{HS}}^{2} d s\right]
$$

and therefore $\left\{\mathcal{L} \widetilde{\Phi}_{n}\right\}_{n \in \mathbb{N}}$ is a sequence of simple processes approximating $\mathcal{L} \Phi$. Hence, by definition of stochastic integration, we find

$$
\int_{0}^{t} \mathcal{L} \Phi(s) d L(s)=\lim _{n \rightarrow \infty} \int_{0}^{t} \mathcal{L} \widetilde{\Phi}_{n}(s) d L(s) .
$$

As $\mathcal{L}$ is a linear bounded operator, we find for any sequence $\left\{X_{n}\right\}_{n \in \mathbb{N}}$ of square integrable random variables in $\widetilde{\mathcal{H}}$ such that $\mathbb{E}\left[\left|X_{n}-X\right|_{\widetilde{\mathcal{H}}}^{2}\right] \rightarrow 0$ for $X \in \widetilde{\mathcal{H}}$ when $n \rightarrow \infty$, that

$$
\lim _{n \rightarrow \infty} \mathbb{E}\left[\left|\mathcal{L}\left(X_{n}-X\right)\right|_{\mathcal{H}}^{2}\right] \leq\|\mathcal{L}\|_{\text {op }}^{2} \lim _{n \rightarrow \infty} \mathbb{E}\left[\left|X_{n}-X\right|_{\widetilde{\mathcal{H}}}^{2}\right]=0
$$

Therefore $\mathcal{L} X_{n}$ converges to $\mathcal{L} X$ in $L^{2}(\Omega ; \mathcal{H})$. Since, by definition,

$$
\int_{0}^{t} \Phi(s) d L(s)=\lim _{n \rightarrow \infty} \int_{0}^{t} \widetilde{\Phi}_{n}(s) d L(s)
$$

it follows that

$$
\begin{aligned}
\mathcal{L} \int_{0}^{t} \Phi(s) d L(s) & =\mathcal{L} \lim _{n \rightarrow \infty} \int_{0}^{t} \widetilde{\Phi}_{n}(s) d L(s)=\lim _{n \rightarrow \infty} \mathcal{L} \int_{0}^{t} \widetilde{\Phi}_{n}(s) d L(s) \\
& =\lim _{n \rightarrow \infty} \int_{0}^{t} \mathcal{L} \widetilde{\Phi}_{n}(s) d L(s)=\int_{0}^{t} \mathcal{L} \Phi(s) d L(s) .
\end{aligned}
$$

The proposition follows.

As a corollary, we obtain the following result:

Corollary 3.2. Assume that the evaluation map $\delta_{x}: \widetilde{\mathcal{H}} \rightarrow \mathcal{H}$ for $x \geq 0$ defined by $\delta_{x} f=f(x)$ is a bounded linear operator, i.e., $\delta_{x} \in L(\widetilde{\mathcal{H}}, \mathcal{H})$ for any $x \geq 0$. If $Y(0)=0$, then, $X(t)=\delta_{0} Y(t)$ for $Y$ in (3.5). 
Proof. By Prop. 3.1 it follows,

$$
\delta_{0} \int_{0}^{t} \Gamma(t+\cdot, s)(\sigma(s)) d L(s)=\int_{0}^{t} \Gamma(t, s)(\sigma(s)) d L(s),
$$

e.g., that the evaluation map commutes with the stochastic integral.

In the following Section 4 we will develop an iterative scheme for $X$ based on finite difference approximation of the solution $Y$ of the SPDE (3.1).

We next construct an explicit example of a space $\widetilde{\mathcal{H}}$.

3.1. An example of $\widetilde{\mathcal{H}}$. We define the Filipovic space for Hilbert space-valued functions on $\mathbb{R}_{+}$. Our extension follows essentially the steps by Filipovic [17] and rests on fundamental properties of so-called vector-valued functions.

Given a separable Hilbert space $\mathcal{H}$ with norm $|\cdot|_{\mathcal{H}}$ induced by the inner product denoted $(\cdot, \cdot)_{\mathcal{H}}$. Let us recall some basic facts of vector-valued functions that we shall need (see Hunter [19]). First, a function $f: \mathbb{R}_{+} \rightarrow \mathcal{H}$ is Bochner integrable if and only if it is weakly measurable and

$$
\int_{\mathbb{R}_{+}}|f(x)|_{\mathcal{H}} d x<\infty .
$$

Weak measurability means that $x \mapsto(f(x), g)_{\mathcal{H}}: \mathbb{R}_{+} \rightarrow \mathbb{R}$ is measurable for every $g \in \mathcal{H}$. We remark that since $\mathcal{H}$ is a separable Hilbert space, weak measurability is equivalent to strong measurability (see Hunter [19], Thm. 6.16, page 197)]. Let $L_{\text {loc }}^{1}\left(\mathbb{R}_{+} ; \mathcal{H}\right)$ be the space of locally Bochner integrable functions $f: \mathbb{R}_{+} \rightarrow \mathcal{H}$. According to Def. 6.31, page 201 in Hunter [19], a function $f \in L_{\mathrm{loc}}^{1}\left(\mathbb{R}_{+} ; \mathcal{H}\right)$ is said to be weakly differentiable if there exists $f^{\prime} \in L_{\mathrm{loc}}^{1}\left(\mathbb{R}_{+} ; \mathcal{H}\right)$ such that

$$
\int_{\mathbb{R}_{+}} f(x) \phi^{\prime}(x) d x=-\int_{\mathbb{R}_{+}} f^{\prime}(x) \phi(x) d x
$$

for all $\phi \in C_{c}^{\infty}\left(\mathbb{R}_{+}\right)$. The integrals above are understood in the Bochner sense.

We are now ready to define a space of $\mathcal{H}$-valued "smooth" functions.

Definition 3.3. For a non-decreasing function $w \in C^{1}\left(\mathbb{R}_{+}\right)$with $w(0)=1$, define

$$
\mathcal{H}_{w}=\left\{f \in L_{\text {loc }}^{1}\left(\mathbb{R}_{+} ; \mathcal{H}\right) \mid \text { there exists } f^{\prime} \in L_{\text {loc }}^{1}\left(\mathbb{R}_{+} ; \mathcal{H}\right) \text { such that }\|f\|_{w}<\infty\right\}
$$

where

$$
\|f\|_{w}^{2}=|f(0)|_{\mathcal{H}}^{2}+\int_{0}^{\infty} w(x)\left|f^{\prime}(x)\right|_{\mathcal{H}}^{2} d x .
$$

Denote by $\langle\cdot, \cdot\rangle_{w}$ the inner product

$$
\langle f, g\rangle_{w}=(f(0), g(0))_{\mathcal{H}}+\int_{0}^{\infty} w(x)\left(f^{\prime}(x), g^{\prime}(x)\right)_{\mathcal{H}} d x,
$$

for $f, g \in \mathcal{H}_{w}$, and observe that $\|f\|_{w}^{2}=\langle f, f\rangle_{w}$.

Proposition 3.4. $\left(\mathcal{H}_{w},\|\cdot\|_{w}\right)$ is a separable Hilbert space.

Proof. The proof adapts the arguments of Thm. 5.1.1 in Filipovic [17] to Hilbert-valued functions. We include the details here for the convenience of the reader.

Observe that $L^{2}\left(\mathbb{R}_{+} ; \mathcal{H}\right)$ is a Hilbert space, and so is $\mathcal{H} \times L^{2}\left(\mathbb{R}_{+} ; \mathcal{H}\right)$ with norm $\|\cdot\|_{*}^{2}:=|\cdot|_{\mathcal{H}}^{2}+\| \cdot$ $\|_{L^{2}\left(\mathbb{R}_{+} ; \mathcal{H}\right)}^{2}$. Define the linear operator $T: \mathcal{H}_{w} \rightarrow \mathcal{H} \times L^{2}\left(\mathbb{R}_{+} ; \mathcal{H}\right)$ by

$$
T f=\left(f(0), f^{\prime} \sqrt{w}\right) \text {. }
$$

$T$ is isometric, since

$$
\|T f\|_{*}^{2}=|f(0)|_{\mathcal{H}}^{2}+\int_{0}^{\infty}\left|\sqrt{w(x)} f^{\prime}(x)\right|_{\mathcal{H}}^{2} d x=\|f\|_{w}^{2} .
$$

\footnotetext{
${ }^{1}$ Strongly measurable means that $f$ can be approximated by simple functions, that is, $f_{n}=\sum_{j=1}^{n} c_{j} 1_{E_{j}}$, where $\left\{E_{j}\right\}_{j \in \mathbb{N}} \subset$ $\mathcal{B}_{\mathbb{R}_{+}}$and $\left\{c_{j}\right\}_{j \in \mathbb{N}} \subset \mathcal{H}$, such that $\left|f(x)-f_{n}(x)\right|_{\mathcal{H}} \rightarrow 0$, a.e. for $x \in \mathbb{R}_{+}$when $n \rightarrow \infty$.
} 
We claim that its inverse is the operator $S: \mathcal{H} \times L^{2}\left(\mathbb{R}_{+} ; \mathcal{H}\right) \rightarrow \mathcal{H}_{w}$ defined as

$$
S(u, h)(x):=u+\int_{0}^{x} h(y) w^{-1 / 2}(y) d y .
$$

First, since $h \in L^{2}\left(\mathbb{R}_{+} ; \mathcal{H}\right)$ and $w^{-1 / 2}(y) \leq 1$ due to $w$ being non-decreasing and $w(0)=1$, we find that the integral is well-defined in the Bochner sense. It holds,

$$
T(S(u, h))=\left(S(u, h)(0), S(u, h)^{\prime} \sqrt{w}\right)=\left(u, h w^{-1 / 2} w^{1 / 2}\right)=(u, h),
$$

where we have applied Thms. 6.32 (page 201) and 6.35 (page 203) in Hunter [19]. Furthermore,

$S(T f)(x)=S\left(f(0), f^{\prime} \sqrt{w}\right)(x)=f(0)+\int_{0}^{x} f^{\prime}(y) \sqrt{w}(y) w^{-1 / 2}(y) d y=f(0)+\int_{0}^{x} f^{\prime}(y) d y=f(x)$,

where we used Thm 6.35 (page 203) in Hunter [19] in the last equality. Hence, $S=T^{-1}$, and $\mathcal{H}_{w}$ is isomorphic to the Hilbert space $\mathcal{H} \times L^{2}\left(\mathbb{R}_{+} ; \mathcal{H}\right)$ implying that $\mathcal{H}_{w}$ is a complete inner product space, i.e., a Hilbert space.

$\mathcal{H}$ is assumed separable, which means that for any $f \in L^{2}\left(\mathbb{R}_{+} ; \mathcal{H}\right)$

$$
f(x)=\sum_{k=1}^{\infty}\left\langle f(x), e_{k}\right\rangle_{\mathcal{H}} e_{k}
$$

for a.e. $x \in \mathbb{R}_{+}$for the ONB $\left\{e_{k}\right\}_{k \in \mathbb{N}} \subset \mathcal{H}$. We have that $f_{k}:=\left\langle f(\cdot), e_{k}\right\rangle_{\mathcal{H}} \in L^{2}\left(\mathbb{R}_{+} ; \mathbb{R}\right)$ since by Schwartz' inequality

$$
\int_{0}^{\infty}\left|f_{k}(x)\right|^{2} d x=\int_{0}^{\infty}\left|\left\langle f(x), e_{k}\right\rangle_{\mathcal{H}}\right|^{2} d x \leq \int_{0}^{\infty}|f(x)|_{\mathcal{H}}^{2} d x\left|e_{k}\right|_{\mathcal{H}}^{2}=\int_{0}^{\infty}|f(x)|_{\mathcal{H}}^{2} d x<\infty
$$

But since $L^{2}\left(\mathbb{R}_{+} ; \mathbb{R}\right)$ is separable, we find for an ONB $\left\{h_{n}\right\}_{n \in \mathbb{N}} \subset L^{2}\left(\mathbb{R}_{+} ; \mathbb{R}\right)$

$$
f_{k}(x)=\sum_{n=1}^{\infty}\left(f_{k}, h_{n}\right)_{L^{2}} h_{n}(x) .
$$

But then $\left\{h_{n} \otimes e_{k}\right\}_{n, k \in \mathbb{N}}$ is an ONB of $L^{2}\left(\mathbb{R}_{+} ; \mathcal{H}\right)$. This shows that $L^{2}\left(\mathbb{R}_{+} ; \mathcal{H}\right)$ is separable, and hence $\mathcal{H} \times L^{2}\left(\mathbb{R}_{+} ; \mathcal{H}\right)$ is separable as well. By the isomorphism $T$, we can therefore conclude the separability of $\mathcal{H}_{w}$. The proof is complete.

The next Lemma provides us with a fundamental theorem of calculus on $\mathcal{H}_{w}$ :

Lemma 3.5. Assume $w^{-1} \in L^{1}\left(\mathbb{R}_{+}\right)$. Then for any $f \in \mathcal{H}_{w}, f^{\prime} \in L^{1}\left(\mathbb{R}_{+} ; \mathcal{H}\right),\left\|f^{\prime}\right\|_{L^{1}\left(\mathbb{R}_{+} ; \mathcal{H}\right)} \leq c\|f\|_{w}$, and

$$
f(x+t)-f(x)=\int_{x}^{x+t} f^{\prime}(y) d y,
$$

for every $x \in \mathbb{R}_{+}$and $t \geq 0$. The constant $c$ is given by $c^{2}:=\int_{0}^{\infty} w^{-1}(x) d x$.

Proof. For $f \in \mathcal{H}_{w}$, we find by the Cauchy-Schwartz inequality,

$$
\begin{aligned}
\int_{0}^{\infty}\left|f^{\prime}(x)\right|_{\mathcal{H}} d x & =\int_{0}^{\infty} w^{-1 / 2}(x) w^{1 / 2}(x)\left|f^{\prime}(x)\right|_{\mathcal{H}} d x \\
& \leq\left(\int_{0}^{\infty} w^{-1}(x) d x\right)^{1 / 2}\left(\int_{0}^{\infty} w(x)\left|f^{\prime}(x)\right|_{\mathcal{H}}^{2} d x\right)^{1 / 2} \\
& \leq\left(\int_{0}^{\infty} w^{-1}(x) d x\right)^{1 / 2}\|f\|_{w}<\infty .
\end{aligned}
$$

Hence, $f^{\prime} \in L^{1}\left(\mathbb{R}_{+} ; \mathcal{H}\right)$ and the norm estimate follows. But then, by Thm. 6.35 (page 203) in Hunter [19 yields the fundamental theorem of calculus. 
This result also tells us that any element of $\mathcal{H}_{w}$ is absolutely continuous, and, in particular, continuous. Introduce now the shift semigroup $\left(\mathcal{S}_{t}\right)_{t \geq 0}$ on $\mathcal{H}_{w}$ defined as

$$
\mathcal{S}_{t} f:=f(\cdot+t) \text {. }
$$

The next Lemma shows uniform boundedness of $\mathcal{S}_{t}$ on $\mathcal{H}_{w}$.

Lemma 3.6. Suppose that $w^{-1} \in L^{1}\left(\mathbb{R}_{+}\right)$, Then $\mathcal{S}_{t}$ is uniformly bounded with $\left\|\mathcal{S}_{t}\right\|_{o p} \leq \sqrt{2\left(1+c^{2}\right)}$. Here, $c$ is the positive constant defined in Lemma 3.5

Proof. Since, by Lemma 3.5 and Thm. 6.32 in Hunter [19], $\left(\mathcal{S}_{t} f\right)^{\prime}=f^{\prime}(\cdot+t)$, we find that

$$
\begin{aligned}
\left\|\mathcal{S}_{t} f\right\|_{w}^{2} & =|f(t)|_{\mathcal{H}}^{2}+\int_{0}^{\infty} w(x)\left|f^{\prime}(x+t)\right|_{\mathcal{H}}^{2} d x \\
& \leq\left|f(0)+\int_{0}^{t} f^{\prime}(y) d y\right|_{\mathcal{H}}^{2}+\int_{t}^{\infty} w(y-t)\left|f^{\prime}(y)\right|_{\mathcal{H}}^{2} d y \\
& \leq 2|f(0)|_{\mathcal{H}}^{2}+2\left|\int_{0}^{t} f^{\prime}(y) d y\right|_{\mathcal{H}}^{2}+\int_{0}^{\infty} w(y)\left|f^{\prime}(y)\right|_{\mathcal{H}}^{2} d y \\
& \leq 2|f(0)|_{\mathcal{H}}^{2}+2\left(\int_{0}^{t}\left|f^{\prime}(y)\right|_{\mathcal{H}} d y\right)^{2}+\int_{0}^{\infty} w(y)\left|f^{\prime}(y)\right|_{\mathcal{H}}^{2} d y
\end{aligned}
$$

In the first inequality we applied Lemma 3.5 while in the second we applied an elementary inequality and the monotonicity of $w$. Finally, in the last estimation step we used the norm inequality for Bochner integrals. Hence, again appealing to the monotonicity of $w$,

$$
\left\|\mathcal{S}_{t} f\right\|_{w}^{2} \leq 2\left(1+c^{2}\right)\|f\|_{w}^{2}
$$

and the proof is complete.

Next, we study continuity properties of the shift semigroup $\mathcal{S}_{t}$. To this end, let

$$
\mathcal{D}:=\left\{f \in \mathcal{H}_{w} \mid f^{\prime} \in \mathcal{H}_{w}\right\},
$$

where we note that $\operatorname{Dom}\left(\partial_{x}\right)=\mathcal{D}$ and $\partial_{x}$ being the derivative operator.

Lemma 3.7. If $w^{-1} \in L^{1}\left(\mathbb{R}_{+}\right)$, the shift operator $\mathcal{S}_{t}$ is strongly continuous on $\mathcal{H}_{w}$.

Proof. We first show strong continuity on $\mathcal{D}$ defined in 3.8 . Indeed, for $f \in \mathcal{H}$ we have by the Lemma 3.5 above

$$
f(t)-f(0)=\int_{0}^{t} f^{\prime}(y) d y .
$$

Moreover, if $f^{\prime} \in \mathcal{H}_{w}$, then the same Lemma yields,

$$
f^{\prime}(x+t)-f^{\prime}(x)=\int_{x}^{x+t} f^{\prime \prime}(y) d y=t \int_{0}^{1} f^{\prime \prime}(x+s t) d s .
$$

Also, we have that $\mathcal{S}_{t} f=f(\cdot+t) \in \mathcal{H}_{w}$ is weakly differentiable (see proof of Lemma 3.5). Thus, for $f \in \mathcal{D}$ we find from the the norm inequality for Bochner integrals and Cauchy-Schwartz' inequality,

$$
\begin{aligned}
\left\|\mathcal{S}_{t} f-f\right\|_{w}^{2} & =|f(t)-f(0)|_{\mathcal{H}}^{2}+\int_{0}^{\infty} w(x)\left|f^{\prime}(x+t)-f^{\prime}(x)\right|_{\mathcal{H}}^{2} d x \\
& =\left|\int_{0}^{t} f^{\prime}(y) d y\right|_{\mathcal{H}}^{2}+\int_{0}^{\infty} w(x) t^{2}\left|\int_{0}^{1} f^{\prime \prime}(x+s t) d s\right|_{\mathcal{H}}^{2} d x \\
& \leq\left(\int_{0}^{t}\left|f^{\prime}(y)\right|_{\mathcal{H}}^{2} d y\right)^{2}+t^{2} \int_{0}^{\infty} w(x)\left(\int_{0}^{1}\left|f^{\prime \prime}(x+s t)\right|_{\mathcal{H}}^{2} d s\right)^{2} d x \\
& \leq\left(\int_{0}^{t}\left|f^{\prime}(y)\right|_{\mathcal{H}}^{2} d y\right)^{2}+t^{2} \int_{0}^{\infty} \int_{0}^{1} w(x)\left|f^{\prime \prime}(x+s t)\right|_{\mathcal{H}}^{4} d s d x \\
& \leq\left(\int_{0}^{t}\left|f^{\prime}(y)\right|_{\mathcal{H}}^{2} d y\right)^{2}+t^{2} \int_{0}^{1}\left\|\mathcal{S}_{s t} f^{\prime}\right\|_{w}^{4} d s .
\end{aligned}
$$


The second integral is finite as $\mathcal{S}_{t}$ is a uniformly bounded operator on $\mathcal{H}_{w}$ from Lemma 3.5 Thus, letting $t \downarrow 0$, we get that $\left\|\mathcal{S}_{t} f-f\right\|_{w} \rightarrow 0$, showing strong continuity on $\mathcal{D}$.

By appealing to a density argument for $\mathcal{D}$ in $\mathcal{H}_{w}$, we can conclude that $\mathcal{S}_{t}$ is strongly continuous on $\mathcal{H}_{w}$ : Introduce the subspace (following Filipovic [17], page 77)

$$
\mathcal{D}_{0}=\left\{f \in C^{2}\left(\mathbb{R}_{+} ; \mathcal{H}\right) \mid f^{\prime} \in C_{c}^{1}\left(\mathbb{R}_{+} ; \mathcal{H}\right)\right\}
$$

where $C^{2}\left(\mathbb{R}_{+} ; \mathcal{H}\right)$ denotes the twice continuously strongly differentiable functions and $C_{c}^{1}\left(\mathbb{R}_{+} ; \mathcal{H}\right)$ functions with compact support being once continuously strongly differentiable. Prop. 6.29 in Hunter [19] ensures that $C_{c}^{1}\left(\mathbb{R}_{+} ; \mathcal{H}\right)$ is dense in $L^{2}\left(\mathbb{R}_{+} ; \mathcal{H}\right)$. For $f \in \mathcal{H}_{w}$, let $\left\{h_{n}\right\}_{n} \subset C_{c}^{1}\left(\mathbb{R}_{+} ; \mathcal{H}\right)$ be an approximating sequence of $f^{\prime} \sqrt{w} \in L^{2}\left(\mathbb{R}_{+} ; \mathcal{H}\right)$. Define $f_{n}:=T^{-1}\left(f(0), h_{n}\right)$ for the operator $T$ defined in (3.6). We have that $f_{n} \in \mathcal{D}_{0}$ and $\left\|f_{n}-f\right\|_{w} \rightarrow 0$ as $n \rightarrow \infty$ because $T$ is an isomorphism (see proof of Prop. 3.4). This shows that $\mathcal{D}_{0}$ is dense in $\mathcal{H}_{w}$.

Thus, for $f, g \in \mathcal{H}_{w}$, the triangle inequality along with the uniform boundedness of $\mathcal{S}_{t}$, yield,

$$
\begin{aligned}
\left\|\mathcal{S}_{t} f-f\right\|_{w} & \leq\left\|\mathcal{S}_{t}(f-g)\right\|_{w}+\left\|\mathcal{S}_{t} g-g\right\|_{w}+\|g-f\|_{w} \\
& \leq \sqrt{2\left(1+c^{2}\right)}\|f-g\|_{w}+\|f-g\|_{w}+\left\|\mathcal{S}_{t} g-g\right\|_{w} .
\end{aligned}
$$

But, since $\mathcal{D}_{0}$ is dense in $\mathcal{H}_{w}$, we choose $g \in \mathcal{D}_{0}$ such that $\|f-g\|_{w} \leq \epsilon / 2\left(1+\sqrt{2\left(1+c^{2}\right)}\right.$. By strong continuity of $\mathcal{S}_{t}$ on $\mathcal{D}$ we choose $t$ such that $\left\|\mathcal{S}_{t} g-g\right\|_{w} \leq \epsilon / 2$. Then, $\mathcal{S}_{t}$ is strongly continuous on $\mathcal{H}_{w}$. The proof is complete.

We conclude that $\mathcal{S}_{t}$ is a $C_{0}$-semigroup on $\mathcal{H}_{w}$ with a generator $\partial_{x}$ being defined on $\mathcal{D}$, a dense subset of $\mathcal{H}_{w}$.

Introduce the evaluation map $\delta_{x} ; \mathcal{H}_{w} \rightarrow \mathcal{H}$ for $x \in \mathbb{R}_{+}$as $\delta_{x} f:=f(x)$ for $f \in \mathcal{H}_{w}$. We prove that $\delta_{x}$ is a bounded linear operator:

Lemma 3.8. Suppose that $w^{-1} \in L^{1}\left(\mathbb{R}_{+}\right)$. Then $\left|\delta_{x} f\right|_{\mathcal{H}} \leq K\|f\|_{w}$ for a positive constant $K$ given by $K^{2}=2 \max \left(1, \int_{0}^{\infty} w^{-1}(y) d y\right)$.

Proof. For $f \in \mathcal{H}_{w}$ it holds by Lemma 3.5 that

$$
\delta_{x} f=f(x)=f(0)+\int_{0}^{x} f^{\prime}(y) d y .
$$

But then by Bochner's norm inequality and Cauchy-Schwartz' inequality,

$$
\begin{aligned}
|f(x)|_{\mathcal{H}}^{2} & \leq 2|f(0)|_{\mathcal{H}}^{2}+2\left|\int_{0}^{x} f^{\prime}(y) d y\right|_{\mathcal{H}}^{2} \\
& \leq 2|f(0)|_{\mathcal{H}}^{2}+2\left(\int_{0}^{x}\left|f^{\prime}(y)\right| \mathcal{H} d y\right)^{2} \\
& \leq 2|f(0)|_{\mathcal{H}}^{2}+2 \int_{0}^{\infty} w^{-1}(y) d y \int_{0}^{\infty} w(y)\left|f^{\prime}(y)\right|_{\mathcal{H}}^{2} d y
\end{aligned}
$$

This concludes the proof.

We end this Subsection with some results on linear functionals on $\mathcal{H}$ and $\mathcal{H}_{w}$. To this end, let $H_{w}$ be the classical Filipovic space (which can be obtained by selecting $\mathcal{H}=\mathbb{R}$ in the definition of $\mathcal{H}_{w}$ above). The norm is denoted by $|\cdot| w$. We have the following proposition:

Proposition 3.9. For $\mathcal{L} \in \mathcal{H}^{*}$ and $g \in \mathcal{H}_{w}$, the real-valued function $x \mapsto \mathcal{L}(g(x))$ on $\mathbb{R}_{+}$is an element of $H_{w}$.

Proof. Recall that if $g \in \mathcal{H}_{w}$, then $g(x) \in \mathcal{H}$ for any $x \in \mathbb{R}_{+}$, and thus $\mathcal{L}(g(\cdot))$ is a real-valued measurable function on $\mathbb{R}_{+}$which is locally integrable. As $g \in \mathcal{H}_{w}$ it is weakly differentiable,

$$
g(x)=g(0)+\int_{0}^{x} g^{\prime}(y) d y
$$


and by properties of the Bochner integral

$$
\mathcal{L}(g(x))=\mathcal{L}(g(0))+\int_{0}^{x} \mathcal{L}\left(g^{\prime}(y)\right) d y .
$$

Hence, $x \mapsto \mathcal{L}(g(x))$ is weakly differentiable and $\partial_{x}(\mathcal{L}(g(x)))=\mathcal{L}\left(g^{\prime}(x)\right)$, for $\partial_{x}$ being the differential operator. Thus,

$$
\begin{aligned}
|\mathcal{L}(g(\cdot))|_{w}^{2} & =|\mathcal{L}(g(0))|^{2}+\int_{0}^{\infty} w(y)\left|\mathcal{L}\left(g^{\prime}(y)\right)\right|^{2} d y \\
& \leq\|\mathcal{L}\|_{\text {op }}^{2}|g(0)|_{\mathcal{H}}^{2}+\|\mathcal{L}\|_{\text {op }}^{2} \int_{0}^{\infty} w(y)\left|g^{\prime}(y)\right|_{\mathcal{H}}^{2} d y \\
& =\|\mathcal{L}\|_{\text {op }}^{2}|g|_{\mathcal{H}}^{2}<\infty .
\end{aligned}
$$

The result follows.

Note that we can write $\mathcal{L}(g(x))=\mathcal{L} \circ \delta_{x}(g)$, and that $\mathcal{L} \circ \delta_{x} \in \mathcal{H}_{w}^{*}$, whenever $\mathcal{L} \in \mathcal{H}^{*}$. This means that there exists a unique $\ell_{x} \in \mathcal{H}_{w}$ such that

$$
\mathcal{L}(g(x))=\mathcal{L} \circ \delta_{x}(g)=\left\langle g, \ell_{x}\right\rangle_{w} .
$$

We can characterize $\ell_{x}$ :

Proposition 3.10. Assume $w^{-1} \in L^{1}\left(\mathbb{R}_{+}\right)$. It holds $\mathcal{L}(g(x))=\left\langle g, \ell_{x}\right\rangle_{w}$ for $\ell_{x}(\cdot) \in \mathcal{H}_{w}$ where

$$
\ell_{x}(\cdot)=\mathcal{L}^{*}\left(h_{x}(\cdot)\right)
$$

for $y \mapsto h_{x}(y)=1+\int_{0}^{x \wedge y} w^{-1}(z) d z \in H_{w}$.

Proof. From Lemma 5.3.1 in Filipovic[17],

$$
\mathcal{L}(g(x))=\bar{\delta}_{x}(\mathcal{L}(g(\cdot)))=\left(\mathcal{L}(g(\cdot)), h_{x}\right)_{w}
$$

where $\bar{\delta}_{x}$ is the evaluation map on $H_{w}$. Hence,

$$
\begin{aligned}
\mathcal{L}(g(x)) & =\left(\mathcal{L}(g(\cdot)), h_{x}\right)_{w} \\
& =\mathcal{L}(g(0)) 1+\int_{0}^{\infty} w(y) \mathcal{L}\left(g^{\prime}(y)\right) h_{x}^{\prime}(y) d y \\
& =\left(g(0), \mathcal{L}^{*} 1\right)_{\mathcal{H}}+\int_{0}^{\infty} w(y)\left(g^{\prime}(y), \mathcal{L}^{*}\left(h_{x}^{\prime}(y)\right)\right)_{\mathcal{H}} d y .
\end{aligned}
$$

We find that $\ell_{x}^{\prime}(y)=\mathcal{L}^{*}\left(h_{x}^{\prime}(y)\right)$ by linearity of $\mathcal{L}^{*}$ and the fundamental theorem of calculus. Noting that $h_{x}(0)=1$, the proof follows.

\section{A FINITE DIFFERENCE SCHEME}

This section presents a finite difference scheme for approximating solutions of a slightly generalized version of the hyperbolic SPDE 3.1. More specifically, we consider the hyperbolic SPDE set in $\widetilde{\mathcal{H}}$

$$
d Y(t)=\partial_{\xi} Y(t) d t+\beta(t) d L(t),
$$

with given initial value $Y(0)=Y_{0} \in \widetilde{\mathcal{H}}$. Here, $\beta(t) \in L(\mathcal{V}, \widetilde{\mathcal{H}})$ is predictable and such that

$$
\mathbb{E}\left[\int_{0}^{t}\left\|\beta(s) \mathcal{Q}^{1 / 2}\right\|_{\mathrm{HS}}^{2} d s\right]<\infty .
$$

For the special case of Hambit processes, we choose $\beta(t)=\Gamma(t+\cdot, t)(\sigma(t))$. However, in this section, we simplify the notation by considering a general stochastic integrand $\beta$. Suppose in addition that

$$
\mathbb{E}\left[\int_{0}^{t}\left\|\mathcal{S}_{t-s} \beta(s) \mathcal{Q}^{1 / 2}\right\|_{\mathrm{HS}}^{2} d s\right]<\infty
$$


then by Peszat and Zabczyk [20, Ch. 6], the SPDE (4.1) possesses a mild solution

$$
Y(t)=\mathcal{S}_{t} Y_{0}+\int_{0}^{t} \mathcal{S}_{t-s} \beta(s) d L(s) .
$$

In what follows, we can easily include a drift in the SPDE above, but we refrain from doing so reduce notation and technicalities.

Let $\Delta x>0$ and $\Delta t>0$ denote the discrete steps in space and time respectively, and set $t_{n}=n \Delta t$, $x_{j}=j \Delta x$ for $n=0, \ldots, N$ and $j=0, \ldots, J$ for some $J, N \in \mathbb{N}$. We aim at introducing an approximation $\widetilde{Y}^{n}$ of $Y$ at time $t_{n}$ of the form

$$
\tilde{Y}^{n}=\sum_{j=0}^{J-1}\left\{\frac{x-x_{j}}{\Delta x}\left(y_{j+1}^{n}-y_{j}^{n}\right)+y_{j}^{n}\right\} \mathbf{1}_{\left[x_{j}, x_{j+1}\right)}(\cdot),
$$

for $x \leq x_{J}$ and $\tilde{Y}^{n}(x)=y_{J}^{n}$ for $x>x_{J}$. Here, $\left\{y_{j}^{n}\right\}_{j=0, \ldots J} \subset \mathcal{H}$. We assume that $\tilde{Y}^{n} \in \tilde{\mathcal{H}}$, and remark that in the case $\widetilde{\mathcal{H}}=\mathcal{H}_{w}$ this assumption holds since the weak derivative of $\tilde{Y}^{n}$ in that case is piecewise constant and zero outside $x>x_{J}$. It is convenient to think of

$$
y_{j}^{n} \approx \delta_{j \Delta x} Y(n \Delta t),
$$

that is, $\delta_{x_{j}} \tilde{Y}^{n}$ approximates the sampled solution of (4.1) at the point $(n \Delta t, j \Delta x)$. Here, we recall the evaluation operator $\delta_{x} \in L(\widetilde{\mathcal{H}}, \mathcal{H})$ introduced in the previous section. For the initial value $Y_{0}$, we introduce the approximation

$$
\widetilde{Y}_{0}:=\sum_{j=0}^{J-1}\left\{\frac{x-x_{j}}{\Delta x}\left(\delta_{x_{j+1}} Y_{0}-\delta_{x_{j}} Y_{0}\right)+\delta_{x_{j}} Y_{0}\right\} \mathbf{1}_{\left[x_{j}, x_{j+1}\right)}(\cdot),
$$

for $x \leq x_{J}$ and $\widetilde{Y}_{0}(x)=\delta_{x_{J}} Y_{0}$ for $x>x_{J}$. This is indeed a linear interpolation of $Y_{0}$ as an element in $\widetilde{\mathcal{H}}$. We assume $\widetilde{Y}_{0} \in \widetilde{\mathcal{H}}$, and obviously let $y_{j}^{0}:=\delta_{x_{j}} \widetilde{Y}_{0}=\delta_{x_{j}} Y_{0}$. Since $\beta(t) \in L(\mathcal{V}, \widetilde{\mathcal{H}}), \delta_{x} \beta(t) \in L(\mathcal{V}, \mathcal{H})$. As we shall see, we need a particular approximation of $\beta(t)$, denoted by $\widetilde{\beta}(t)$ and given as (for $x \leq x_{J}$ )

$$
\tilde{\beta}(t)=\sum_{j=0}^{J-1}\left\{\frac{x-x_{j}}{\Delta x}\left(\delta_{x_{j+1}} \beta(t)-\delta_{x_{j}} \beta(t)\right)+\delta_{x_{j}} \beta(t)\right\} \mathbf{1}_{\left[x_{j}, x_{j+1}\right)}(\cdot),
$$

and $\widetilde{\beta}(t)(x)=\delta_{x_{J}} \beta(t)$ for $x>x_{J}$. Thus, we sample the operator $\beta(t) \in L(\mathcal{V}, \widetilde{\mathcal{H}})$ into a linear combination of operators $\delta_{x_{j}} \beta(t) \in L(\mathcal{V}, \mathcal{H}), j=0, \ldots, J$. We see that $x \mapsto \widetilde{\beta}(t)(x)$ is a function from $\mathbb{R}_{+}$into $L(\mathcal{V}, \mathcal{H})$. We therefore define $\widetilde{\beta}(t) \in L(\mathcal{V}, \widetilde{\mathcal{H}})$ by

$$
\widetilde{\beta}(t)(f)=\sum_{j=0}^{J-1}\left\{\frac{x-x_{j}}{\Delta x}\left(\delta_{x_{j+1}} \beta(t)(f)-\delta_{x_{j}} \beta(t)(f)\right)+\delta_{x_{j}} \beta(t)(f)\right\} \mathbf{1}_{\left[x_{j}, x_{j+1}\right)}(\cdot),
$$

for $f \in \mathcal{V}$, with $x \leq x_{J}$. When $x>x_{J}$, we let $\widetilde{\beta}(t)(f)(x)=\delta_{x_{J}} \beta(t)(f)$. Since $\delta_{x_{j}} \beta(t)(f) \in \mathcal{H}, \widetilde{\beta}(t)(f)$ is a function from $\mathbb{R}_{+}$into $\mathcal{H}$. We assume that $\widetilde{\beta}(t)(f) \in \widetilde{\mathcal{H}}$ from now on, and remark that when $\widetilde{\mathcal{H}}=\widetilde{\mathcal{H}}_{w}$, this assumption is fulfilled since we have a piecewise constant weak derivative which is zero outside $x_{J}$.

To derive a recursive scheme for $y_{j}^{n}$ in $n$, we use finite difference approximations of the SPDE (4.1), thus using $d Y(t) \approx Y(t+\Delta t)-Y(t), d t \approx \Delta t, d L(t) \approx L(t+\Delta t)-L(t)$ and $\partial_{\xi} Y(t) \approx(Y(t)(\cdot+$ $\Delta x)-Y(t)) / \Delta x$ in 4.1) to find the finite difference scheme

$$
y_{j}^{n+1}=\lambda y_{j+1}^{n}+(1-\lambda) y_{j}^{n}+\beta_{j}^{n}\left(\Delta L^{n}\right),
$$

where $\lambda=\Delta t / \Delta x, \beta_{j}^{n}=\delta_{x_{j}} \beta\left(t_{n}\right)$ and $\Delta L^{n}=L\left(t_{n+1}\right)-L\left(t_{n}\right)$.

We note that the finite difference scheme (4.6) is a Hilbert space generalization of a scheme proposed and analysed by Benth and Eyjolfsson [9]. In that paper a numerical approximation of real-valued VMV processes based on a scheme for a hyperbolic SPDE was introduced, analogous to the case we study here. Our infinite dimensional approach and analysis that follows are inspired by Benth and Eyjolfsson [9]. Notice that the information in the finite difference scheme in (4.6) flows to the left as time progresses. Hence, for a given time $n \Delta t$, the scheme will provide values for $y_{j}^{n+1}, j=0, \ldots, J-1$ for the next time 
step. As we wish to study our approximation $\widetilde{Y}^{n}$ for $n=0,1, \ldots N$ and $x \leq x_{J}$, we can adjust our finite differencing to be made for suitably large choices of grid points in space $x$ initially, so that at terminal time $N \Delta t$ we have a computation of $y_{j}^{N}$ for all $j=0, \ldots, J$. Indeed, this is the same as letting $J$ be depending on the time step $n$. We refrain from going into technical details on the practicalities here, but refer to Benth and Eyjolfsson [9] for more discussion.

As in the case of a finite difference scheme for the standard advection partial differential equation, one needs some constraints on the discrete steps, i.e. $(\Delta x, \Delta t)$, to guarantee its stability. The stability condition of Courant, Friedrichs, and Lewy (the CFL condition, see [15]) is needed to ensure the stability of our finite difference scheme 4.6. In our case this translates into the necessary constraint

$$
\Delta t \leq \Delta x
$$

which we assume to hold.

Given our Hilbert space $\widetilde{\mathcal{H}}$ of $\mathcal{H}$-valued functions on $\mathbb{R}_{+}$it will be convenient for our analysis to define the following family of bounded linear operators on $\widetilde{\mathcal{H}}$. Given positive $\Delta x>0$ and $\Delta t>0$ corresponding to the steps of the finite difference scheme in space and time respectively consider the family $\left\{\mathcal{T}_{\Delta x, \Delta t}\right\}_{\Delta x>0, \Delta t>0}$ which is defined by

$$
\mathcal{T}_{\Delta x, \Delta t}=\mathcal{I}+\Delta t \frac{\mathcal{S}_{\Delta x}-\mathcal{I}}{\Delta x}
$$

for all $\Delta x>0, \Delta t>0$, where $\mathcal{I}$ denotes the identity operator on $\widetilde{\mathcal{H}}$.

Lemma 4.1. For given steps $\Delta x>0$ in space and $\Delta t>0$ in time, $\widetilde{Y}^{n}$ admits the representation

$$
\tilde{Y}^{n}=\mathcal{T}^{n} \widetilde{Y}_{0}+\sum_{i=0}^{n-1} \mathcal{T}^{n-1-i} \widetilde{\beta}^{i}\left(\Delta L^{i}\right)
$$

for $n=0, \ldots, N$. Here, $\mathcal{T}:=\mathcal{T}_{\Delta x, \Delta t}$ is defined by [4.8, and we use the conventions that $\mathcal{T}^{n}=\mathcal{T}^{\circ n}$ denotes the composition of the operator $\mathcal{T}$ with itself $n$ times, and $\mathcal{T}^{0}=\mathcal{I}$.

Proof. We prove the result by induction. It clearly holds for $n=0$, since then $\tilde{Y}^{0}=\tilde{Y}_{0}=\mathcal{T}^{0} \widetilde{Y}_{0}$. Next, suppose that it holds for $n \in \mathbb{N}$. Assume that $x \in\left[x_{j}, x_{j+1}\right)$ for a given $j \in \mathbb{N}, j \leq J$. Then, $x+\Delta x \in\left[x_{j+1}, x_{j+2}\right)$, and we find

$$
\begin{aligned}
\delta_{x} \mathcal{T} \tilde{Y}^{n} & =\delta_{x} \mathcal{I} \tilde{Y}^{n}+\lambda \delta_{x}\left(\mathcal{S}_{\Delta x}-\mathcal{I}\right) \tilde{Y}^{n} \\
& =y_{j}^{n}+\lambda\left(y_{j+1}^{n}-y_{j}^{n}\right)+\frac{x-x_{j}}{\Delta x}\left(y_{j+1}^{n}+\lambda\left(y_{j+2}^{n}-y_{j+1}^{n}\right)\right)-\frac{x-x_{j}}{\Delta x}\left(y_{j}^{n}+\lambda\left(y_{j+1}^{n}-y_{j}^{n}\right)\right) .
\end{aligned}
$$

But by the finite difference scheme (4.6), it follows

$$
\begin{aligned}
\delta_{x} \mathcal{T} \tilde{Y}^{n}= & y_{j}^{n+1}-\beta_{j}^{n}\left(\Delta L^{n}\right)+\frac{x-x_{j}}{\Delta x}\left(y_{j+1}^{n+1}-\beta_{j+1}^{n}\left(\Delta L^{n}\right)\right)-\frac{x-x_{j}}{\Delta x}\left(y_{j}^{n+1}-\beta_{j}^{n}\left(\Delta L^{n}\right)\right) \\
= & y_{j}^{n+1}+\frac{x-x_{j}}{\Delta x}\left(y_{j+1}^{n+1}-y_{j}^{n+1}\right) \\
& \quad-\left(\beta_{j}^{n}\left(\Delta L^{n}\right)+\frac{x-x_{j}}{\Delta x}\left(\beta_{j+1}^{n}\left(\Delta L^{n}\right)-\beta_{j}^{n}\left(\Delta L^{n}\right)\right)\right) .
\end{aligned}
$$

By invoking the definition of $\widetilde{\beta}(t)$ and noting that $x$ can be chosen arbitrary,

$$
\widetilde{Y}^{n+1}=\mathcal{T} \tilde{Y}^{n}+\widetilde{\beta}^{n}\left(\Delta L^{n}\right)
$$

From the induction hypothesis, we then find

$$
\tilde{Y}^{n+1}=\mathcal{T}^{n+1} \widetilde{Y}_{0}+\mathcal{T} \sum_{i=0}^{n-1} \mathcal{T}^{n-1-i} \widetilde{\beta}^{i}\left(\Delta L^{i}\right)+\widetilde{\beta}^{n}\left(\Delta L^{n}\right)=\mathcal{T}^{n+1} \widetilde{Y}_{0}+\sum_{i=0}^{n} \mathcal{T}^{n-i} \widetilde{\beta}^{i}\left(\Delta L^{i}\right) .
$$

This completes the proof. 
The above lemma characterizes the finite difference scheme (4.6) for a given discretization as the sum of two entities which, under appropriate conditions, will converge to their corresponding parts in the mild solution of (4.1) as we consider finer and finer partitions in time and space. More precisely we will employ the fact that the composed operator $\mathcal{T}^{n}$, where $\mathcal{T}=\mathcal{T}_{\Delta x, \Delta t}$ is defined by (4.8), converges to the left shift operator $\mathcal{S}_{t_{n}}$ as we consider finer and finer partitions in first time and then space.

Let us take a closer look on the family (4.8) of operators. The following lemma will be employed later for proving a convergence result on the finite difference scheme.

Lemma 4.2. Suppose $\zeta$ is an $\widetilde{\mathcal{H}}$-valued random variable satisfying the Lipschitz condition

$$
\mathbb{E}\left[\left\|\left(\mathcal{S}_{x} \zeta-\mathcal{S}_{y} \zeta\right) \mathcal{Q}^{1 / 2}\right\|_{H S}^{2}\right] \leq C|x-y|^{2}
$$

for all $x, y \geq 0$ where $C>0$ is a constant. Then

$$
\mathbb{E}\left[\left\|\left(\mathcal{T}^{m} \zeta-\mathcal{S}_{t} \zeta\right) \mathcal{Q}^{1 / 2}\right\|_{H S}^{2}\right] \leq C t(\Delta x-\Delta t)
$$

where $\mathcal{T}$ is defined in (4.8) with $\Delta t=t / m$ and $\Delta t \leq \Delta x$, for all $x \geq 0, t>0$ and $m \geq 1$.

Proof. Let $\lambda=\Delta t / \Delta x$ and suppose first that $\lambda=1$, then clearly $\mathcal{T}=\mathcal{S}_{\Delta x}$ and $\mathcal{T}^{m}=\mathcal{S}_{t}$. Now suppose that $\lambda<1$, and observe that by the binomial theorem it holds that

$$
\mathcal{T}^{m} \zeta=(1-\lambda)^{m}\left(\mathcal{I}+\frac{\lambda}{1-\lambda} \mathcal{S}_{\Delta x}\right)^{m} \zeta=\sum_{k=0}^{m}\left(\begin{array}{c}
m \\
k
\end{array}\right) \lambda^{k}(1-\lambda)^{m-k} \mathcal{S}_{k \Delta x} \zeta
$$

It follows by the triangle inequality that

$$
\begin{aligned}
\left\|\left(\mathcal{T}^{m} \zeta-\mathcal{S}_{t} \zeta\right) \mathcal{Q}^{1 / 2}\right\|_{\mathrm{HS}}^{2} & =\left\|\left(\sum_{k=0}^{m}\left(\begin{array}{c}
m \\
k
\end{array}\right) \lambda^{k}(1-\lambda)^{m-k}\left(\mathcal{S}_{k \Delta x} \zeta-\mathcal{S}_{t} \zeta\right)\right) \mathcal{Q}^{1 / 2}\right\|_{\mathrm{HS}}^{2} \\
& \leq\left|\sum_{k=0}^{m}\left(\begin{array}{c}
m \\
k
\end{array}\right) \lambda^{k}(1-\lambda)^{m-k}\left\|\left(\mathcal{S}_{k \Delta x} \zeta-\mathcal{S}_{t} \zeta\right) \mathcal{Q}^{1 / 2}\right\|_{\mathrm{HS}}\right|^{2} \\
& \leq \sum_{k=0}^{m}\left(\begin{array}{c}
m \\
k
\end{array}\right) \lambda^{k}(1-\lambda)^{m-k}\left\|\left(\mathcal{S}_{k \Delta x} \zeta-\mathcal{S}_{t} \zeta\right) \mathcal{Q}^{1 / 2}\right\|_{\mathrm{HS}}^{2} .
\end{aligned}
$$

In the last step we applied the Cauchy-Schwarz inequality. Finally, we employ the Lipschitz condition on $\zeta$ to derive,

$$
\mathbb{E}\left[\left\|\left(\mathcal{T}^{m} \zeta-\mathcal{S}_{t} \zeta\right) \mathcal{Q}^{1 / 2}\right\|_{\mathrm{HS}}^{2}\right] \leq C \sum_{k=0}^{m}\left(\begin{array}{c}
m \\
k
\end{array}\right) \lambda^{k}(1-\lambda)^{m-k}|k \Delta x-t|^{2} .
$$

Observing that a binomial random variable $Z$, with parameters $(m, \lambda)$ has expected value $m \lambda$ and variance $m \lambda(1-\lambda)$, it is easy to deduce that the random variable $\Delta x Z$ has expected value $t$ and variance $t(\Delta x-\Delta t)$. Hence,

$$
\sum_{k=0}^{m}\left(\begin{array}{c}
m \\
k
\end{array}\right) \lambda^{k}(1-\lambda)^{m-k}|k \Delta x-t|^{2}=t(\Delta x-\Delta t) .
$$

This concludes the proof.

We can apply the same type of argument to derive the error induced by approximating $\mathcal{S}_{t} Y_{0}$ by $\mathcal{T}^{m} \widetilde{Y}_{0}$ :

Lemma 4.3. Assume for $x, y \in \mathbb{R}_{+}$that $\left|\mathcal{S}_{x} Y_{0}-\mathcal{S}_{y} Y_{0}\right|_{\widetilde{\mathcal{H}}} \leq C_{0}|x-y|$ for some positive constant $C_{0}$. Then,

$$
\left|\mathcal{T}^{m} \widetilde{Y}_{0}-\mathcal{S}_{t} Y_{0}\right|_{\tilde{\mathcal{H}}} \leq C_{0} \sqrt{t(\Delta x-\Delta t)}+\sup _{u \leq t}\left\|\mathcal{S}_{u}\right\|_{o p}\left|\tilde{Y}_{0}-Y_{0}\right|_{\tilde{\mathcal{H}}}
$$

where $\mathcal{T}$ is defined in (4.8) with $\Delta t=t / m$ and $\Delta t \leq \Delta x$, for all $x \geq 0, t>0$ and $m \geq 1$. 
Proof. By the triangle inequality

$$
\left|\mathcal{T}^{m} \widetilde{Y}_{0}-\mathcal{S}_{t} Y_{0}\right|_{\widetilde{\mathcal{H}}} \leq\left|\mathcal{T}^{m} \tilde{Y}_{0}-\mathcal{T}^{m} Y_{0}\right|_{\widetilde{\mathcal{H}}}+\left|\mathcal{T}^{m} Y_{0}-\mathcal{S}_{t} Y_{0}\right|_{\widetilde{\mathcal{H}}}
$$

For the second term on the right hand side, using the Lipschitz assumption on $Y_{0}$, we can repeat the argument in the proof Lemma 4.2 for the norm $|\cdot|_{\widetilde{\mathcal{H}}}$ instead of $\|\cdot\|_{\text {HS }}$ to obtain

$$
\left|\mathcal{T}^{m} Y_{0}-\mathcal{S}_{t} Y_{0}\right|_{\tilde{\mathcal{H}}} \leq C_{0} \sqrt{t(\Delta x-\Delta t)}
$$

For the first term, we find

$$
\left|\mathcal{T}^{m} \widetilde{Y}_{0}-\mathcal{T}^{m} Y_{0}\right|_{\widetilde{\mathcal{H}}} \leq\left\|\mathcal{T}^{m}\right\|_{\text {op }}\left|\widetilde{Y}_{0}-Y_{0}\right|_{\widetilde{\mathcal{H}}}
$$

Now suppose first that $\lambda=1$, then clearly $\mathcal{T}=\mathcal{S}_{\Delta x}$ and $\mathcal{T}^{m}=\mathcal{S}_{t}$. If however $\lambda<1$, then

$$
\left\|\mathcal{T}^{m}\right\|_{\text {op }}=\sup \left\{\left|\mathcal{T}^{m} f\right|_{\widetilde{\mathcal{H}}}: f \in \widetilde{\mathcal{H}},|f|_{\widetilde{\mathcal{H}}}=1\right\},
$$

and we may apply the binomial theorem to obtain

$$
\mathcal{T}^{m} f=(1-\lambda)^{m}\left(\mathcal{I}+\frac{\lambda}{1-\lambda} \mathcal{S}_{\Delta x}\right)^{m} f=\sum_{k=0}^{m}\left(\begin{array}{c}
m \\
k
\end{array}\right) \lambda^{k}(1-\lambda)^{m-k} \mathcal{S}_{k \Delta x} f,
$$

so it follows by the triangle inequality that

$$
\left\|\mathcal{T}^{m}\right\|_{\text {op }} \leq \max _{0 \leq k \leq m}\left\|\mathcal{S}_{k \Delta x}\right\|_{\text {op }} \leq \sup _{u \leq t}\left\|\mathcal{S}_{u}\right\|_{\text {op }}
$$

This completes the proof.

In general the operator norm of a $C_{0}$-semigroup grows at most exponentially with time, so that we find $\sup _{u \leq t}\left\|\mathcal{S}_{u}\right\|_{\text {op }} \leq c_{1} \exp \left(c_{2} t\right)$ for positive constants $c_{1}, c_{2}$. If $\widetilde{\mathcal{H}}=\mathcal{H}_{w}$ with $w^{-1} \in L^{1}\left(\mathbb{R}_{+}\right)$, the shift semigroup $\mathcal{S}_{t}$ is uniformly bounded by Lemma 3.6, and moreover $\sup _{u \leq t}\left\|\mathcal{S}_{u}\right\|_{\text {op }} \leq \sqrt{2\left(1+c^{2}\right)}$ for $c^{2}=\int_{0}^{\infty} w^{-1}(x) d x$.

Proposition 4.4. Assume that for $s, u, x, y \in \mathbb{R}_{+}$,

and

$$
\begin{gathered}
\left|\mathcal{S}_{x} Y_{0}-\mathcal{S}_{y} Y_{0}\right|_{\widetilde{\mathcal{H}}} \leq C_{0}|x-y| \\
\mathbb{E}\left[\left\|\left(\mathcal{S}_{x} \beta(s)-\mathcal{S}_{y} \beta(s)\right) \mathcal{Q}^{1 / 2}\right\|_{H S}^{2}\right] \leq C|x-y|^{2},
\end{gathered}
$$

$$
\mathbb{E}\left[\left\|(\beta(s)-\beta(u)) \mathcal{Q}^{1 / 2}\right\|_{H S}^{2}\right] \leq C|s-u|^{2},
$$

for positive constants $C_{0}, C$. Then, for $t_{n}=n \Delta t$ and $x_{j}=j \Delta x, n, j \geq 0$, it holds that

$$
\begin{array}{rl}
\mathbb{E}\left[\left|\widetilde{Y}^{N}-Y\left(t_{N}\right)\right|_{\widetilde{\mathcal{H}}}^{2}\right] \leq 4 & t\left(C_{0}^{2}+2 C t\right)(\Delta x-\Delta t)+8 C t\left(1+\frac{1}{3} \sup _{u \leq t}\left\|\mathcal{S}_{u}\right\|_{o p}^{2}\right)(\Delta t)^{2} \\
& +4 \sup _{u \leq t}\left\|\mathcal{S}_{u}\right\|_{o p}^{2} \mathbb{E}\left[\left|\widetilde{Y}_{0}-Y_{0}\right|_{\widetilde{\mathcal{H}}}^{2}\right] \\
& +8 t \sup _{u \leq t}\left\|\mathcal{S}_{u}\right\|_{o p}^{2} \max _{0 \leq i \leq N-1} \mathbb{E}\left[\left\|\left(\widetilde{\beta}^{i}-\beta^{i}\right) \mathcal{Q}^{1 / 2}\right\|_{H S}^{2}\right] .
\end{array}
$$

where $\Delta t=t / N$ and $\Delta t \leq \Delta x$, for all $x \geq 0, t>0$ and $N \geq 1$.

Proof. Since $L$ is square integrable it holds by the Itô isometry that

$$
\begin{aligned}
\mathbb{E}\left[\left|\sum_{i=0}^{N-1} \mathcal{T}^{N-1-i} \widetilde{\beta}^{i}\left(\Delta L^{i}\right)-\sum_{i=0}^{N-1} \mathcal{S}_{t-t_{i+1}} \beta^{i}\left(\Delta L^{i}\right)\right|_{\widetilde{\mathcal{H}}}^{2}\right] \\
=\mathbb{E}\left[\left|\int_{0}^{t}\left(\sum_{i=0}^{N-1}\left(\mathcal{T}^{N-1-i} \widetilde{\beta}^{i}-\mathcal{S}_{t-t_{i+1}} \beta^{i}\right) \mathbf{1}_{\left[t_{i}, t_{i+1}\right)}(s)\right) d L(s)\right|_{\widetilde{\mathcal{H}}}^{2}\right] \\
=\mathbb{E}\left[\int_{0}^{t}\left\|\sum_{i=0}^{N-1}\left(\mathcal{T}^{N-1-i} \widetilde{\beta}^{i}-\mathcal{S}_{t-t_{i+1}} \beta^{i}\right) \mathbf{1}_{\left[t_{i}, t_{i+1}\right)}(s) \mathcal{Q}^{1 / 2}\right\|_{\mathrm{HS}}^{2} d s\right]
\end{aligned}
$$


REPRESENTATION AND APPROXIMATION OF AMBIT FIELDS IN HILBERT SPACE

$$
=\sum_{i=0}^{N-1} \mathbb{E}\left[\left\|\left(\mathcal{T}^{N-1-i} \widetilde{\beta}^{i}-\mathcal{S}_{t-t_{i+1}} \beta^{i}\right) \mathcal{Q}^{1 / 2}\right\|_{\mathrm{HS}}^{2}\right] \Delta t
$$

Adding and subtracting $\mathcal{T}^{N-1-i} \beta^{i}$ and applying the elementary inequality $(x+y)^{2} \leq 2 x^{2}+2 y^{2}$ yields,

$$
\begin{aligned}
\mathbb{E}\left[\left|\sum_{i=0}^{N-1} \mathcal{T}^{N-1-i} \widetilde{\beta}^{i}\left(\Delta L^{i}\right)-\sum_{i=0}^{N-1} \mathcal{S}_{t-t_{i+1}} \beta^{i}\left(\Delta L^{i}\right)\right|_{\widetilde{\mathcal{H}}}^{2}\right] \\
\leq 2 \sum_{i=0}^{N-1} \mathbb{E}\left[\left\|\left(\mathcal{T}^{N-1-i} \widetilde{\beta}^{i}-\mathcal{T}^{N-1-i} \beta^{i}\right) \mathcal{Q}^{1 / 2}\right\|_{\mathrm{HS}}^{2}\right] \Delta t \\
+2 \sum_{i=0}^{N-1} \mathbb{E}\left[\left\|\left(\mathcal{T}^{N-1-i} \beta^{i}-\mathcal{S}_{t-t_{i+1}} \beta^{i}\right) \mathcal{Q}^{1 / 2}\right\|_{\mathrm{HS}}^{2}\right] \Delta t
\end{aligned}
$$

We estimate the second term by appealing to Lemma 4.2, whereas the first term is majorized by using the inequality (4.10). Hence,

$$
\begin{aligned}
& \mathbb{E}\left[\left|\sum_{i=0}^{N-1} \mathcal{T}^{N-1-i} \widetilde{\beta}^{i}\left(\Delta L^{i}\right)-\sum_{i=0}^{N-1} \mathcal{S}_{t-t_{i+1}} \beta^{i}\left(\Delta L^{i}\right)\right|_{\widetilde{\mathcal{H}}}^{2}\right] \\
& \leq 2 \sup _{u \leq t}\left\|\mathcal{S}_{u}\right\|_{\mathrm{op}}^{2} \sum_{i=0}^{N-1} \mathbb{E}\left[\left\|\left(\widetilde{\beta}^{i}-\beta^{i}\right) \mathcal{Q}^{1 / 2}\right\|_{\mathrm{HS}}^{2}\right] \Delta t+2 C t^{2}(\Delta x-\Delta t) \\
& \leq 2 t \sup _{u \leq t}\left\|\mathcal{S}_{u}\right\|_{\mathrm{op}}^{2} \max _{0 \leq i \leq N-1} \mathbb{E}\left[\left\|\left(\widetilde{\beta}^{i}-\beta^{i}\right) \mathcal{Q}^{1 / 2}\right\|_{\mathrm{HS}}^{2}\right]+2 C t^{2}(\Delta x-\Delta t) .
\end{aligned}
$$

Furthermore by Lipschitz continuity of $\beta$ and the Itô isometry,

$$
\begin{aligned}
\mathbb{E} & {\left[\left|\sum_{i=0}^{N-1} \mathcal{S}_{t-t_{i+1}} \beta^{i}\left(\Delta L^{i}\right)-\int_{0}^{t} \mathcal{S}_{t-s} \beta(s) d L(s)\right|_{\tilde{\mathcal{H}}}^{2}\right] } \\
& =\mathbb{E}\left[\left|\int_{0}^{t}\left(\sum_{i=0}^{N-1} \mathcal{S}_{t-t_{i+1}} \beta^{i} 1_{\left[t_{i}, t_{i+1}\right)}(s)-\mathcal{S}_{t-s} \beta(s)\right) d L(s)\right|_{\widetilde{\mathcal{H}}}^{2}\right] \\
& =\sum_{i=0}^{N-1} \mathbb{E}\left[\int_{t_{i}}^{t_{i+1}}\left\|\left(\mathcal{S}_{t-t_{i+1}} \beta^{i}-\mathcal{S}_{t-s} \beta(s)\right) \mathcal{Q}^{1 / 2}\right\|_{\mathrm{HS}}^{2} d s\right] \\
& \leq 2 \sum_{i=0}^{N-1} \mathbb{E}\left[\int_{t_{i}}^{t_{i+1}}\left(\left\|\left(\mathcal{S}_{t-t_{i+1}} \beta^{i}-\mathcal{S}_{t-s} \beta^{i}\right) \mathcal{Q}^{1 / 2}\right\|_{\mathrm{HS}}^{2}+\left\|\mathcal{S}_{t-s}\left(\beta^{i}-\beta(s)\right) \mathcal{Q}^{1 / 2}\right\|_{\mathrm{HS}}^{2}\right)\right] d s \\
& \leq 2 t C(\Delta t)^{2}+2 \sup _{u \leq t}\left\|\mathcal{S}_{u}\right\|_{\mathrm{op}}^{2} \sum_{i=0}^{N-1} \int_{t_{i}}^{t_{i+1}} \mathbb{E}\left[\left\|\left(\beta\left(t_{i}\right)-\beta(s)\right) \mathcal{Q}^{1 / 2}\right\|_{\mathrm{HS}}^{2}\right] d s \\
& \leq 2 C t\left(1+\frac{1}{3} \sup _{u \leq t}\left\|\mathcal{S}_{u}\right\|_{\mathrm{op}}^{2}\right)(\Delta t)^{2} .
\end{aligned}
$$

Putting the above inequalities together, we obtain

$$
\begin{aligned}
& \mathbb{E}\left[\left|\sum_{i=0}^{N-1} \mathcal{T}^{N-1-i} \widetilde{\beta}^{i}\left(\Delta L^{i}\right)-\int_{0}^{t} \mathcal{S}_{t-s} \beta(s) d L(s)\right|_{\widetilde{\mathcal{H}}}^{2}\right] \\
& \leq 2 \mathbb{E}\left[\left|\sum_{i=0}^{N-1} \mathcal{S}_{t-t_{i+1}} \beta^{i}\left(\Delta L^{i}\right)-\int_{0}^{t} \mathcal{S}_{t-s} \beta(s) d L(s)\right|_{\widetilde{\mathcal{H}}}^{2}\right]
\end{aligned}
$$




$$
\begin{aligned}
&++2 \mathbb{E}\left[\left|\sum_{i=0}^{N-1} \mathcal{T}^{N-1-i} \widetilde{\beta}^{i}\left(\Delta L^{i}\right)-\sum_{i=0}^{N-1} \mathcal{S}_{t-t_{i+1}} \beta^{i}\left(\Delta L^{i}\right)\right|_{\widetilde{\mathcal{H}}}^{2}\right] \\
& \leq 4 t \sup _{u \leq t}\left\|\mathcal{S}_{u}\right\|_{\mathrm{op}}^{2} \max _{0 \leq i \leq N-1} \mathbb{E}\left[\left\|\left(\widetilde{\beta}^{i}-\beta^{i}\right) \mathcal{Q}^{1 / 2}\right\|_{\mathrm{HS}}^{2}\right] \\
&+4 C t\left(1+\frac{1}{3} \sup _{u \leq t}\left\|\mathcal{S}_{u}\right\|_{\mathrm{op}}^{2}\right)(\Delta t)^{2}+4 C t^{2}(\Delta x-\Delta t) .
\end{aligned}
$$

The proof is completed after invoking Lemma 4.3 .

Recall that $\beta(t):=\Gamma(t+\cdot, t)(\sigma(t)$ in the case of a Hambit field, for which we see that $\beta(s)-\beta(u)=$ $\mathcal{S}_{s-u} \beta(u)-\mathcal{S}_{0} \beta(u)$ for $s \geq u \geq 0$. Hence, the two Lipschitz conditions on $\beta$ in the Proposition above collapse into one, namely

$$
\mathbb{E}\left[\left\|\Gamma(s+x, s)(\sigma(s))-\Gamma(s+y, s)(\sigma(s)) Q^{1 / 2}\right\|_{H S}^{2}\right] \leq C|x-y|^{2},
$$

for all $x, y, s \in \mathbb{R}_{+}$. Thus, if the operator $\Gamma$ is Lipschitz continuous in its first argument, the conditions on $\beta$ are fulfilled. The condition on $Y_{0}$ is trivially satisfied for Hambit fields as $Y_{0}=0$ in that case.

As we have already touched upon it is not trivial to express a given Hambit field in terms of a certain finite set of vectors in $\mathcal{H}$. It is however the case according to Proposition 2.6 that for given ONB's in the Hilbert spaces $\mathcal{U}, \mathcal{V}$ and $\mathcal{H}$, a general Hambit field can be represented as a countable sum of real-valued VMV processes scaled by the ONB vectors in $\mathcal{H}$. Although it is difficult to say anything in general about the rate at which that sum converges, it is clear that it can be truncated, and thus our finite difference scheme (4.6) can be implemented at least in an approximative manner, for a given Hambit field which fulfills the conditions stated in Proposition 2.6

Now let us elucidate what the above convergence result means for the Hilbert space $\widetilde{\mathcal{H}}=\mathcal{H}_{w}$, which we introduced in the previous section. Note that,

$$
\left\|\left(\widetilde{\beta}^{i}-\beta^{i}\right) \mathcal{Q}^{1 / 2}\right\|_{\mathrm{HS}} \leq\left\|\mathcal{Q}^{1 / 2}\right\|_{\mathrm{HS}}\left\|\widetilde{\beta}^{i}-\beta^{i}\right\|_{\mathrm{op}}=\left\|\mathcal{Q}^{1 / 2}\right\|_{\mathrm{HS}} \sup _{|f| \mathcal{V}=1}\left|\left(\widetilde{\beta}^{i}-\beta^{i}\right)(f)\right|_{\widetilde{\mathcal{H}}} \cdot
$$

Therefore, the convergence of

depends on the convergence of

$$
\max _{0 \leq i \leq N-1} \mathbb{E}\left[\left\|\left(\widetilde{\beta}^{i}-\beta^{i}\right) \mathcal{Q}^{1 / 2}\right\|_{\mathrm{HS}}^{2}\right]
$$

$$
\left\|\left(\widetilde{\beta}^{i}-\beta^{i}\right)(f)\right\|_{w}=\left|\left(\widetilde{\beta}^{i}-\beta^{i}\right) f(0)\right|_{\mathcal{H}}^{2}+\int_{0}^{\infty} w(x)\left|\left(\widetilde{\beta}^{i}-\beta^{i}\right)(f)^{\prime}(x)\right|_{\mathcal{H}}^{2} d x,
$$

in $L^{2}(\Omega)$, where $|f|_{\mathcal{V}}=1$, as we consider finer and finer partitions. We remark that if $f \in \mathcal{V}$ and $x \in\left[x_{j}, x_{j+1}\right)$, then we may express the weak derivative above as

$$
\left(\widetilde{\beta}^{i}-\beta^{i}\right)(f)^{\prime}(x)=\frac{\delta_{x_{j+1}}-\delta_{x_{j}}}{\Delta x} \beta^{i} f-\beta^{i} f^{\prime}(x) .
$$

That is, the right hand side is equal to the difference between a $\mathcal{H}$-valued finite difference approximation and its corresponding weak derivative evaluated at $x \in\left[x_{j}, x_{j+1}\right)$. So the convergence of the scheme depends on the convergence of the above finite difference approximation in $\mathcal{H}$.

\section{REFERENCES}

[1] O. E. Barndorff-Nielsen, F. E., Benth and A. Veraart (2011). Ambit processes and stochastic partial differential equations. In Advanced Mathematical Methods for Finance, G. Di Nunno and B. Øksendal (eds.), Springer-Verlag Berlin Heidelberg, Ch. 2, pp. 35-74.

[2] O. E. Barndorff-Nielsen, F. E., Benth and A. Veraart (2013). Modelling energy spot prices by volatility modulated Lévy-driven Volterra processes. Bernoulli, 19(3), pp. 803-845.

[3] O. E. Barndorff-Nielsen, F. E., Benth and A. Veraart (2014). Modelling electricity futures by ambit fields. Adv. Appl. Prob., 46, pp. 719-745.

[4] O. E. Barndorff-Nielsen, F. E., Benth and A. Veraart (2015). Recent advances in ambit stochastics with a view towards tempospatial stochastic volatility/intermittency. Banach Center Publications, Vol. 104, pp. 25-60.

[5] O. E. Barndorff-Nielsen and J. Schmiegel (2004), Lévy-based tempo-spatial modelling; with applications to turbulence. Uspekhi Mat. NAUK, 59, pp. 65-91. 
[6] Barndorff-Nielsen, O. E., and Shephard, N. (2001). Non-Gaussian Ornstein-Uhlenbeck based models and some of their uses in financial economics. J. Royal Statist. Soc.: Series B, 63(2), pp. 167-241.

[7] Benth, F. E., and Šaltytė Benth, J. (2012). Modelling and Pricing in Financial Markets for Weather Derivatives. World Scientific, Singapore.

[8] Benth, F. E., Šaltytė Benth, J., and Koekebakker, S. (2008). Stochastic Modelling of Electricity and Related Markets. World Scientific, Singapore.

[9] F.E. Benth, and H. Eyjolfsson, (2015+). Simulation of volatility modulated Volterra processes using hyperbolic stochastic partial differential equations. To appear in Bernoulli.

[10] Benth, F.E., Klüppelberg, C., Müller, G., and Vos, L. (2014). Futures pricing in electricity markets based on stable CARMA models. Energy Econ., 44, pp. 392-406.

[11] Benth, F. E., and Krühner, P. (2014). Representation of infinite dimensional forward price models in commodity markets. Commun. Math. Stat., 2, pp. 47-106.

[12] Benth, F. E., Rüdiger, B., and Süss, A. (2015). Ornstein-Uhlenbeck processes in Hilbert space with non-Gaussian stochastic volatility. Submitted manuscript. Available on arXiv:1506.07245

[13] Brockwell, P. J. (2001). Lévy-driven CARMA processes. Ann. Inst. Math., 53, pp. 113-124.

[14] Corcuera, J. M., Farkas G., Schoutens, W., Valkeila. E. (2012). A short rate model using ambit processes. In Malliavin Calculus and Stochastic Analysis. A Festschrift in Honor of David Nualart. Springer Proceedings in Mathematics \& Statistics, Volume 34, pp 525-553, Springer Verlag.

[15] Courant, R., Friedrichs, O. \& Lewy, H. (1928), Über die partiellen Differenzengleichungen der mathematischen Physik. Mathematische Annalen, 100, pp. 32-74.

[16] Eyjolfsson, H. (2015). Approximating ambit fields via Fourier methods. Stochastics, 87(5), pp. 885-917.

[17] Filipovic, D. (2001). Consistency Problems for Heath-Jarrow-Morton Interest Rate Models. Springer Verlag, Berlin Heidelberg.

[18] Folland, G. B. (1984). Real Analysis. John Wiley \& Sons, New York.

[19] Hunter, J. K (2014). Notes on Partial Differential Equations. Lecture Notes downloaded from https://www.math.ucdavis.edu/ hunter/pdes/pde_notes.pdf (accessed August 31, 2015).

[20] S. Peszat and J. Zabczyk (2007). Stochastic Partial Differential Equations with Lévy Noise. Cambridge University Press, Cambridge.

[21] Protter, Ph. (2005). Stochastic Integration and Differential Equations. Springer Verlag, Berlin Heidelberg.

[22] Vedel Jensen, E., Yr Jonsdottir, K., Schmiegel, J., and Barndorff-Nielsen, O. E. (2007). Spatio-temporal modelling: with a view to biological growth. In Statistical Methods for Spatio-Temporal Systems, Finkenstadt, B., Held, L., and Isham, V. (eds.), pp. 47-76, Chapman-Hall.

[23] Walsh, J. (1984). An introduction to stochastic partial differential equations. In Lecture Notes in Mathematics, Vol 1180, Ecole d'ete de Probabilites de Saint-Flour XIV, Carmona, R., Kesten, H., and Walsh, J. (eds.), Springer Verlag.

(Fred Espen Benth), Department of Mathematics, University of Oslo, P.O. Box 1053, BLindern, N-0316 Oslo, Norway, And, Centre for Advanced Study, Drammens Veien 78, N-0271 Oslo, Norway

E-mail address: fredb@math.uio.no

URL: http://folk.uio.no/fredb/

(Heidar Eyjolfsson), Department of Mathematics, University of Bergen, P.O. Box 7803, N-5020 BerGen, NorWAY.

E-mail address: Heidar.Eyjolfsson@uib.no

URL: http://www.uib.no/en/persons/Heidar.Eyjolfsson 